\title{
Vitamin D levels and deficiency with different occupations: a systematic review
}

\author{
Daniel Sowah ${ }^{1 \dagger}$, Xiangning Fan ${ }^{1 \dagger}$, Liz Dennett $^{2}$, Reidar Hagtvedt ${ }^{3}$ and Sebastian Straube ${ }^{1 *}$ (D)
}

\begin{abstract}
Background: Vitamin D deficiency is prevalent worldwide, but some groups are at greater risk. We aim to evaluate vitamin D levels in different occupations and identify groups vulnerable to vitamin D deficiency.

Methods: An electronic search conducted in Medline, Embase, the Cochrane Central Register of Controlled Trials, and CINAHL Plus with Full Text generated 2505 hits; 71 peer-reviewed articles fulfilled the inclusion criteria. Occupations investigated included outdoor and indoor workers, shiftworkers, lead/smelter workers, coalminers, and healthcare professionals. We calculated the pooled average metabolite level as mean \pm SD; deficiency/insufficiency status was described as $\%$ of the total number of subjects in a given category.
\end{abstract}

Results: Compared to outdoor workers, indoor workers had lower 25-hydroxyvitamin D (25-(OH)D) levels (40. $6 \pm 13.3$ vs. $66.7 \pm 16.7 \mathrm{nmol} / \mathrm{L} ; p<0.0001$ ). Mean $25-(\mathrm{OH}) \mathrm{D}$ levels (in nmol/L) in shiftworkers, lead/smelter workers and coalminers were $33.8 \pm 10.0,77.8 \pm 5.4$ and $56.6 \pm 28.4$, respectively. Vitamin D deficiency $(25-(\mathrm{OH})$ $\mathrm{D}<50 \mathrm{nmol} / \mathrm{L}$ ), was high in shiftworkers (80\%) and indoor workers (78\%) compared to outdoor workers (48\%). Among healthcare professionals, medical residents and healthcare students had the lowest levels of mean 25-(OH) $\mathrm{D}, 44.0 \pm 8.3 \mathrm{nmol} / \mathrm{L}$ and $45.2 \pm 5.5 \mathrm{nmol} / \mathrm{L}$, respectively. The mean $25-(\mathrm{OH}) \mathrm{D}$ level of practising physicians, $55.0 \pm 5$. $8 \mathrm{nmol} / \mathrm{L}$, was significantly different from both medical residents $(p<0.0001)$ and healthcare students $(p<0.0001)$. Nurses and other healthcare employees had $25-(\mathrm{OH}) \mathrm{D}$ levels of $63.4 \pm 4.2 \mathrm{nmol} / \mathrm{L}$ and $63.0 \pm 11.0 \mathrm{nmol} / \mathrm{L}$, respectively, which differed significantly compared to practising physicians $(p=0.01)$, medical residents $(p<0.0001)$ and healthcare students $(p<0.0001)$.

Rates of vitamin D deficiency among healthcare professionals were: healthcare students $72 \%$, medical residents $65 \%$, practising physicians $46 \%$, other healthcare employees $44 \%$, and nurses $43 \%$. Combined rates of vitamin D deficiency or insufficiency $(25-(\mathrm{OH}) \mathrm{D}<75 \mathrm{nmol} / \mathrm{L})$ were very high in all investigated groups.

Potential confounders such as gender and body composition were not consistently reported in the primary studies and were therefore not analyzed. Furthermore, the descriptions of occupational characteristics may be incomplete. These are limitations of our systematic review.

Conclusions: Our review demonstrates that shiftworkers, healthcare workers and indoor workers are at high risk to develop vitamin D deficiency, which may reflect key lifestyle differences (e.g. sunlight exposure). This may help target health promotion and preventive efforts.

Keywords: Vitamin D level, vitamin D deficiency, 25-hydroxyvitamin D (25-(OH)D), occupation, systematic review

\footnotetext{
* Correspondence: straube@ualberta.ca

${ }^{\dagger}$ Equal contributors

'Division of Preventive Medicine, Department of Medicine, University of

Alberta, 5-30 University Terrace, 8303-112 Street, Edmonton, AB T6G 2T4,

Canada

Full list of author information is available at the end of the article
} International License (http://creativecommons.org/licenses/by/4.0/), which permits unrestricted use, distribution, and reproduction in any medium, provided you give appropriate credit to the original author(s) and the source, provide a link to the Creative Commons license, and indicate if changes were made. The Creative Commons Public Domain Dedication waiver (http://creativecommons.org/publicdomain/zero/1.0/) applies to the data made available in this article, unless otherwise stated. 


\section{Background}

Although there has been recent improvement in vitamin D status in the North American population, the prevalence of insufficiency remains high. About 70\%-97\% of Canadians are estimated to demonstrate vitamin D insufficiency [1] and approximately $40 \%$ of the US population are estimated to be vitamin D deficient [2]. While vitamin $\mathrm{D}$ has traditionally been shown to be involved in calcium homeostasis [1] and bone health [2], recent evidence suggests several roles not limited to the musculoskeletal system. An inadequate level of vitamin $\mathrm{D}$ has been linked to a number of diseases including metabolic disorders, autoimmune conditions, psychiatric, respiratory and cardiovascular disorders, and cancers as well as osteoporosis and osteomalacia [2-4]. The widespread systemic effects of vitamin $D$ have been attributed to the ubiquitous expression of vitamin D receptors in various organ systems $[2,5]$.

Vitamin D is synthesized in vivo when solar ultraviolet $B$ (UVB) radiation interacts with the precursor molecule, 7 -dehydrocholesterol, in the skin [2, 6, 7]. Another important source of vitamin D is dietary intake and supplementation, although endogenous production is estimated to account for $90 \%$ of total vitamin D in healthy individuals, and any activity that reduces sunlight exposure will tend to reduce vitamin D levels $[2,8]$. Whether from endogenous production or dietary sources, vitamin D is subsequently transported in the blood (bound to vitamin D-binding protein) to the liver where it is hydroxylated to 25-hydroxyvitamin D (25$(\mathrm{OH}) \mathrm{D})$ [7]. 25- $(\mathrm{OH}) \mathrm{D}$ is further converted to the metabolically active form, $1 \alpha, 25$-dihydroxyvitamin $\mathrm{D}(1 \alpha, 25$ $\left.(\mathrm{OH})_{2} \mathrm{D}\right)$, primarily in the kidneys [9]. In the present study, the term 'vitamin D' was used in the context of status, i.e., deficiency, insufficiency or sufficiency, while $25-(\mathrm{OH}) \mathrm{D}$ referred to serum levels of the metabolite.

Because of its half-life in blood of $\sim 2-5$ weeks, the circulating concentration of $25-(\mathrm{OH}) \mathrm{D}$ has been used as a measure of vitamin D status in individuals [6]. Serum 25$(\mathrm{OH}) \mathrm{D}$ concentrations lower than $50 \mathrm{nmol} / \mathrm{L}(20 \mathrm{ng} / \mathrm{mL})$ appear to be detrimental to bone health [10]; however, optimal serum 25- $(\mathrm{OH}) \mathrm{D}$ concentrations have not been established with respect to other outcomes, and there is lack of agreement on how deficiency should be defined, or how best to conduct population-based screening for vitamin D deficiency [11]. Despite a lack of consensus on optimal levels, it is becoming abundantly evident that vitamin $\mathrm{D}$ deficiency and its associated untoward health outcomes are a worldwide phenomenon $[12,13]$.

As vitamin D synthesis is highly dependent on sunlight, factors and conditions associated with decreased time spent outdoors can be expected to adversely impact vitamin D status. Shiftwork represents work that occurs outside the traditional 9 a.m. to 5 p.m. workday and may include evening or night shift work, with or without rotating shifts. An estimated 28\% of working Canadians [14], $17 \%$ of Americans [15], and $22 \%$ of European workers [16] work outside the traditional 9 a.m. to 5 p.m., Monday through Friday schedule. Shiftwork has been epidemiologically associated with a number of health conditions, including sleep disturbances, cardiovascular disorders, gastrointestinal and digestive problems, and increased cancer risk, among others [17-19]. Little is known about the role of vitamin D deficiency with respect to the adverse health outcomes related to shiftwork, although vitamin D deficiency has been previously postulated as a mechanism of shiftwork-related cancers [20]; shiftworkers can plausibly be expected to have lower serum vitamin $\mathrm{D}$ levels due to reduced exposure to sunlight or altered dietary intake of vitamin D-rich foods. Additionally, other occupational groups (e.g. indoor workers) may be at risk of vitamin D deficiency through this same mechanism of reduced sunlight exposure, and it has been previously noted that there has been comparatively little research into the health of indoor nonindustrial workers [21].

Understanding the levels of vitamin D and prevalence of vitamin $\mathrm{D}$ deficiency in various occupational categories can inform public health attempts to reduce vitamin $\mathrm{D}$ deficiency and ensure improved population health outcomes. The link between vitamin D levels and occupation has previously been explored in the published literature, and low levels of vitamin D have been demonstrated in some occupational groups with expected low exposure to sunlight. For example, a recent study in bakers concluded that vitamin D insufficiency was very common, especially in night workers [22]. A large study from Korea found that the risk of vitamin D deficiency was significantly increased for shift work and office work [23]. An Indonesian study likewise showed that vitamin $\mathrm{D}$ deficiency may occur in women with indoor occupations [24]. In contrast, other evidence confirms that outdoor workers have comparatively high serum vitamin D levels [25-27]. However, the association between occupational factors (e.g. shiftwork, indoor work, work activities) and vitamin D levels is far from clear in the literature, and vitamin D deficiency in working populations does not seem to be entirely explained by sunlight exposure. A Japanese study on shiftworkers comparing serum $25-(\mathrm{OH}) \mathrm{D}$ levels in fixed daytime workers to rotating workers with or without night shift demonstrated no significant differences [28]. Additionally, vitamin D deficiency can be prevalent among certain groups of workers, for example health care workers, even in regions with high sunshine exposure [29].

To our knowledge, there has been no previous systematic attempt to examine the effect of occupation on vitamin D status, or determine the prevalence of vitamin D deficiency in different occupational groups, despite the 
importance of work in most workers' lives. Additionally, we are unaware of any current guidelines on screening for vitamin D deficiency or vitamin D supplementation, which include specific guidance for workers, or risk stratification elements based on occupational factors. The present article aims to provide evidence to address these gaps.

\section{Methods}

The objective of the present study is to investigate serum vitamin D levels, and prevalence of insufficiency and deficiency in different occupational categories to identify groups of workers at particular risk of vitamin D deficiency or insufficiency.

\section{Study eligibility criteria}

We sought observational studies describing measured vitamin $\mathrm{D}$ levels or prevalence of vitamin D deficiency or insufficiency in a working population. We included studies where a distinct group of workers was compared with one or more groups of other workers or nonworking individuals, and studies on students provided they were students of a specific vocation (e.g. healthcare students). Otherwise, we excluded studies on students. We excluded studies performed on subjects in the military, professional athletes, and astronauts, and studies taking place in Antarctica, because they were deemed less relevant to our study objective. We also excluded trials of vitamin D supplementation in working populations. Only peer-reviewed full journal articles were included; we excluded review articles, abstracts and conference proceedings, as well as articles not published in English or German. We included papers in which data were derived from large population based cohort studies in a defined occupational group (e.g. the Nurses' Health Study, Physicians' Health Study, etc.). For case-control studies, we excluded information on cases but included information on controls, as they would arguably have been representative of the study population and were not defined by a disease state.

\section{Search strategy}

Electronic database searches were conducted by a health sciences librarian (LD) in July 2015 and updated in March 2016 in Medline (including in process and other nonindexed citations as well as Medline Daily), Embase, the Cochrane Central Register of Controlled Trials, and CINAHL Plus with Full Text. The searches used an extensive combination of keywords and subject headings for the concepts of vitamin D and occupation to identify relevant studies. Studies that only included participants who were 18 and under or 65 and older were excluded as they were assumed not to be about working populations. Furthermore, studies where the described occupations could not be meaningfully grouped into occupational categories have been excluded from this review. The full version of the electronic search strategies can be found in 'Additional file 1: Search strategies'. Reference lists of retrieved articles and reviews in the field were assessed to identify additional publications of relevance. Search results and fulltext articles were screened independently by two investigators (XF, DS). Wherever there was a disagreement in the selection of relevant articles between the two investigators, the senior investigator (SS) made the final judgement based on the established inclusion/exclusion criteria.

\section{Assessment of study quality and data extraction}

We extracted data on the study ID, number of subjects, location/latitude, measured vitamin D levels (25-hydroxyvitamin D, 25-hydroxyvitamin D2, 25-hydroxyvitamin $\mathrm{D} 3$, and $1 \alpha, 25$-dihydroxyvitamin $\mathrm{D}$ ) and on the prevalence of vitamin $\mathrm{D}$ deficiency and insufficiency. Additionally, we extracted data on season of the year and the assay type from articles where this information was available. Where the latitude of the location of study was not provided but the name of the city or country of study was given, it was obtained from an online tool on the National Aeronautics and Space Administration (NASA) website by entering the city or country name and searching for the respective latitude [30]. The extracted data also included first author, year of publication, study type and occupational group. Data extraction was performed by one investigator and independently verified by a second (XF, DS). Discrepancy between the two investigators was resolved by consulting with the senior investigator (SS).

The quality of the included studies was assessed based on previously employed criteria [12], which included the representativeness (selection of study subjects) of the individual study report, the validity of method used to measure vitamin D levels [31,32], and assay reliability, which pertains to the intra- and inter-assay coefficients of variation of the assay. However, in the present report, representativeness of study participants was not a major concern since serum 25-(OH)D status was assessed in workers of a given occupational category; therefore, we did not include this factor in our evaluation of study quality.

One criterion that we used to assess study quality was whether the authors mentioned the season of the year in which the study was conducted, as there is a seasonal variation in vitamin D levels [4] which is an important confounder when interpreting vitamin D levels. Additionally, a particular study was considered valid if the assay technique to determine vitamin $\mathrm{D}$ levels was in keeping with the International Vitamin D Quality Assessment Scheme [33]. Finally, to be considered reliable, the inter-assay coefficient of variation $(\mathrm{CV})$ must be less than $15 \%$, while the 
intra-assay CV must be below 10\% [12, 33]. A study was considered to be of high quality if all three criteria (reporting on seasonality, validity and reliability) were met; of medium quality when only two of the criteria were satisfied; when only one criterion was met, we considered such a study to be of low quality; and when none of our quality criteria were met, the study was considered to be of very low quality.

\section{Data synthesis}

The mean levels of measured vitamin D metabolites (25$(\mathrm{OH}) \mathrm{D}$ and/or $\left.1 \alpha, 25-(\mathrm{OH})_{2} \mathrm{D}\right)$ were extracted from included studies for each occupational category. Studies where metabolite levels were provided as mean $\pm \mathrm{SD} / \mathrm{SE}$ (standard deviation/standard error) were included in further analysis to compute the overall mean for the particular occupational group. To enable pooling of data from different studies for a particular occupational group, we also standardized the measure of spread by converting SE to SD, where applicable, using the formula, $S D=S E x \sqrt{ }$, where $\mathrm{N}$ is the sample size or number of subjects.

Following conversion to SD, the pooled SD was calculated by combining the individually weighted SD based on the formula below [34]:

$$
\mathrm{S}_{\mathrm{P}}^{2}=\frac{\left(\mathrm{n}_{1}-1\right) \mathrm{SD}_{1}^{2}+\left(\mathrm{n}_{2}-1\right) \mathrm{SD}_{2}^{2}}{\mathrm{n}_{1}+\mathrm{n}_{2}-2}
$$

$S_{\mathrm{B}}^{2}$ pooled variance.

$\mathrm{n}_{1}=$ sample size of group 1 .

$\mathrm{n}_{2}=$ sample size of group 2 .

$\mathrm{SD}_{1}=$ standard deviation of group 1 .

$\mathrm{SD}_{2}=$ standard deviation of group 2 .

Pooled standard error, SEp, was calculated according to the following formula [35]:

$$
S E p=S p \sqrt{\frac{1}{\mathrm{n}_{1}}+\frac{1}{\mathrm{n}_{2}}}
$$

When averages were presented in the study as median \pm interquartile ranges (IQRs), the values were converted into estimated mean \pm SD based on a previously established approach [36-38]. Data were not included in the final meta-analysis if only the median values were provided in the absence of IQRs [39, 40] or geometric means reported without indication of a measure of spread $[41,42]$. Whenever there were three or more studies from an occupational group, which reported data as mean $\pm \mathrm{SD}$, a meta-analysis was conducted on those studies by pooling or combining the means and SDs using the method for combining means and SDs as described in the Cochrane Handbook for Systematic Reviews of Interventions [43]. Based on this approach, the overall mean of each occupational category was calculated by averaging the means of individual studies weighted by the number of subjects of each study.

All units of measurement of vitamin D concentration were standardized to the S.I. units, $\mathrm{nmol} / \mathrm{L}$ for 25$(\mathrm{OH}) \mathrm{D}$ or $\mathrm{pmol} / \mathrm{L}$ for $1 \alpha, 25-(\mathrm{OH})_{2} \mathrm{D}$, by multiplying the imperial unit $(\mathrm{ng} / \mathrm{ml}$ or $\mathrm{pg} / \mathrm{ml}$, respectively) by a factor of 2.5 or 2.4 , respectively [12]. The number of studies reporting on levels of $1 \alpha, 25-(\mathrm{OH})_{2} \mathrm{D}$ were too few to permit quantitative comparison of results obtained between different occupational groups. Results were therefore mainly analyzed and compared relative to the average serum levels of 25-(OH)D computed from each occupational category. To evaluate the differences in the prevalence of vitamin D insufficiency and deficiency between different occupational groups, the proportion of study subjects who were either deficient or insufficient in the relevant groups were compared.

Due to the lack of consensus regarding the level of 25$(\mathrm{OH}) \mathrm{D}$ that constitutes vitamin D deficiency, we adopted the widely employed Endocrine Society's (ES) cut-offs as standard definition to compare the degree of deficiency between different occupational groups. The ES has defined vitamin deficiency as a serum $25-(\mathrm{OH}) \mathrm{D}$ concentration of $<20 \mathrm{ng} / \mathrm{ml} \quad(<50 \mathrm{nmol} / \mathrm{L})$, a serum level between $20 \mathrm{ng} / \mathrm{ml}(50 \mathrm{nmol} / \mathrm{L})$ and $30 \mathrm{ng} / \mathrm{ml}(<75 \mathrm{nmol} /$ L) as insufficiency, and a level $>30 \mathrm{ng} / \mathrm{ml}(>75 \mathrm{nmol} / \mathrm{L})$ as adequate to maintain normal physiological function $[2,44]$. However, when levels of deficiency were provided in the absence of mean $25-(\mathrm{OH}) \mathrm{D}$ level, such studies were not included in further meta-analysis.

We chose the weighted average of the proportions of insufficient and deficient vitamin D status as the baseline for comparison with specific occupational groups. We calculated relative risk (RR) by finding the percentage increase (or decrease) in proportion, compared to this baseline with a $95 \%$ confidence interval (CI).

\section{Statistical analysis}

Data on the average serum 25-(OH)D levels of each occupational group are reported as mean $\pm \mathrm{SD}$. The levels of 25-(OH)D deficiency or combined deficiency/insufficiency of each occupational group are reported as a percentage of the total number of subjects in the given group. Whether the difference in means between occupational categories was statistically significant, was determined with the unpaired Student's t-test. We used a Chi-squared test to determine the significance of differences between proportions of vitamin D deficiency or deficiency/insufficiency between occupational groups. A $p$-value of $<0.05$ was considered statistically significant. The Bonferroni procedure was employed to test whether pairwise differences were statistically significant, while retaining an overall level of significance of $5 \%$. Data were 
extracted and analyzed in Microsoft Excel and $p$-values were estimated with GraphPad software.

\section{Results}

The electronic database search generated 1991 records after deduplication, of which 87 primary studies (all in English) were considered potentially relevant, based on title and abstract screening, and available as full text journal articles. An additional 17 articles were identified by searching reference lists of previously identified articles or reviews. Of this total of 104 articles, 33 were ultimately excluded after review of the full-text article, leaving 71 articles to be included for further quantitative analysis as shown in 'Additional file 2: Study selection'. The total number of subjects for all included studies was at least 53,345 (one study did not report on the number of subjects) and the sample sizes ranged from 4 to 10,646 subjects per study as shown in Table 1 . The overall proportion of participants who were women was 65\%. Publication dates ranged from 1971 to 2016. Based on the latitudes of study locations, the included studies spanned a range of latitudes from $3^{\circ} \mathrm{N}$ (Indonesia) to $64^{\circ} \mathrm{N}$ (Reykjavik, Iceland) in the Northern hemisphere, and $23^{\circ} \mathrm{S}$ (Sao Paulo, Brazil) to $30^{\circ} \mathrm{S}$ (Porto Alegre, Brazil) in the Southern hemisphere (Table 1 and Additional file 3: Figure S4A).

Thirty-five of 71 articles described studies performed in health care workers (physicians, nurses, hospital employees, health sciences or medical students, and other health professionals). Three studies were performed in coal miners, 5 in lead/smelter workers and 6 in shiftworkers. Eleven of the 71 papers described groups of outdoor workers; 19 studies were performed in indoor or office-based workers. Some of the primary studies included subjects of more than one occupational setting and such studies were categorized under more than one occupational group depending on the occupations described by the authors, as shown in Table 1. Each occupational category that we examined had 3 or more primary studies, therefore permitting further quantitative analysis. Studies in which we could not establish a welldefined occupational setting were excluded (Table 1).

We extracted data from the included studies based on season of the year in which the study was conducted, assay type (measure of validity) and intra- and interassay CV (indication of reliability) in order to assess study quality; 'unknown' indicates that a study did not describe the parameter in question (Table 2). As shown in Table 2, 43\% of included studies were of high quality, $37 \%$ were of medium quality, and $20 \%$ were of low quality. Regarding assay types, $40 \%$ of reports employed a radioimmunoassay (RIA) technique to assess serum vitamin D levels, 14\% used a competitive protein binding assay, a chemiluminescence assay technique was employed in $13 \%$ of studies, $11 \%$ assayed vitamin D levels via the high performance liquid chromatography (HPLC) assay, while $4 \%, 2 \%$ and $1 \%$, respectively, employed the enzyme-linked immunosorbent assay (ELISA), liquid chromatography and radioceptor techniques.

\section{Indoor/office and outdoor workers}

We compared vitamin D level and the proportion of workers with deficiency/insufficiency between indoor and outdoor workers. As shown in Fig. 1 (and Additional file 4: Figure S1), the mean vitamin D level was significantly lower in indoor/office workers compared to outdoor workers $(40.6 \pm 13.2 \mathrm{nmol} / \mathrm{L} \quad$ vs. $66.6 \pm 16.7 \mathrm{nmol} / \mathrm{L} ; p<0.0001)$. Figure 2 demonstrates that $78 \%$ of indoor workers were vitamin $\mathrm{D}$ deficient in contrast to only $48 \%$ of outdoor workers who were vitamin D deficient. There was also a statistically significant difference between indoor and outdoor workers in the proportion who were vitamin D deficient or insufficient: 91\% of indoor workers had vitamin D levels below $75 \mathrm{nmol} / \mathrm{L}$ versus $75 \%$ of outdoor workers $(p<0.01)$ (Fig. 2). Indoor workers had significantly elevated RRs of 1.23 (95\% CI: 1.22 to 1.24 ) and 1.24 (95\% CI: 1.22 to 1.25), to develop vitamin D deficiency and insufficiency, respectively (Tables 3 and 4). Outdoor workers had a significantly reduced susceptibility to vitamin D deficiency (RR: 0.77 ; $95 \%$ CI: 0.75 to 0.79 ) but no significantly different risk to develop vitamin D insufficiency (RR: 1.02; 95\% CI: 0.98 to 1.07) (Tables 3 and 4).

To determine the effect of latitude on vitamin D status and deficiency, the latitudes of the various study locations were obtained and plotted against mean vitamin D levels, \% deficiency and \% non-vitamin D sufficient (e.g. deficient or insufficient). On average, at any given latitude, the mean vitamin $\mathrm{D}$ levels of outdoor workers were higher than values seen in indoor workers (Additional file 3: Figure S4B). In general, a higher proportion of indoor workers were vitamin D deficient compared to outdoor workers (Additional file 5: Figure S5B). That vitamin D deficiency or insufficiency was higher in indoor workers relative to outdoor workers was not dependent on study location (Additional file 6: Figure S6B).

\section{Shiftworkers}

Our analysis demonstrated that the impact of shiftwork on vitamin D status was considerable. Of all the occupational categories that were studied, shiftworkers had the lowest average levels of serum vitamin D $(33.8 \pm 10.1 \mathrm{nmol} / \mathrm{L})$ (Fig. 1). About $80 \%$ of shiftworkers had serum vitamin $\mathrm{D}$ levels $\leq 50 \mathrm{nmol} / \mathrm{L}$, indicating vitamin D deficiency (Fig. 2). Of the 6 studies on shiftworkers, only 1 study [45] reported \% vitamin D 


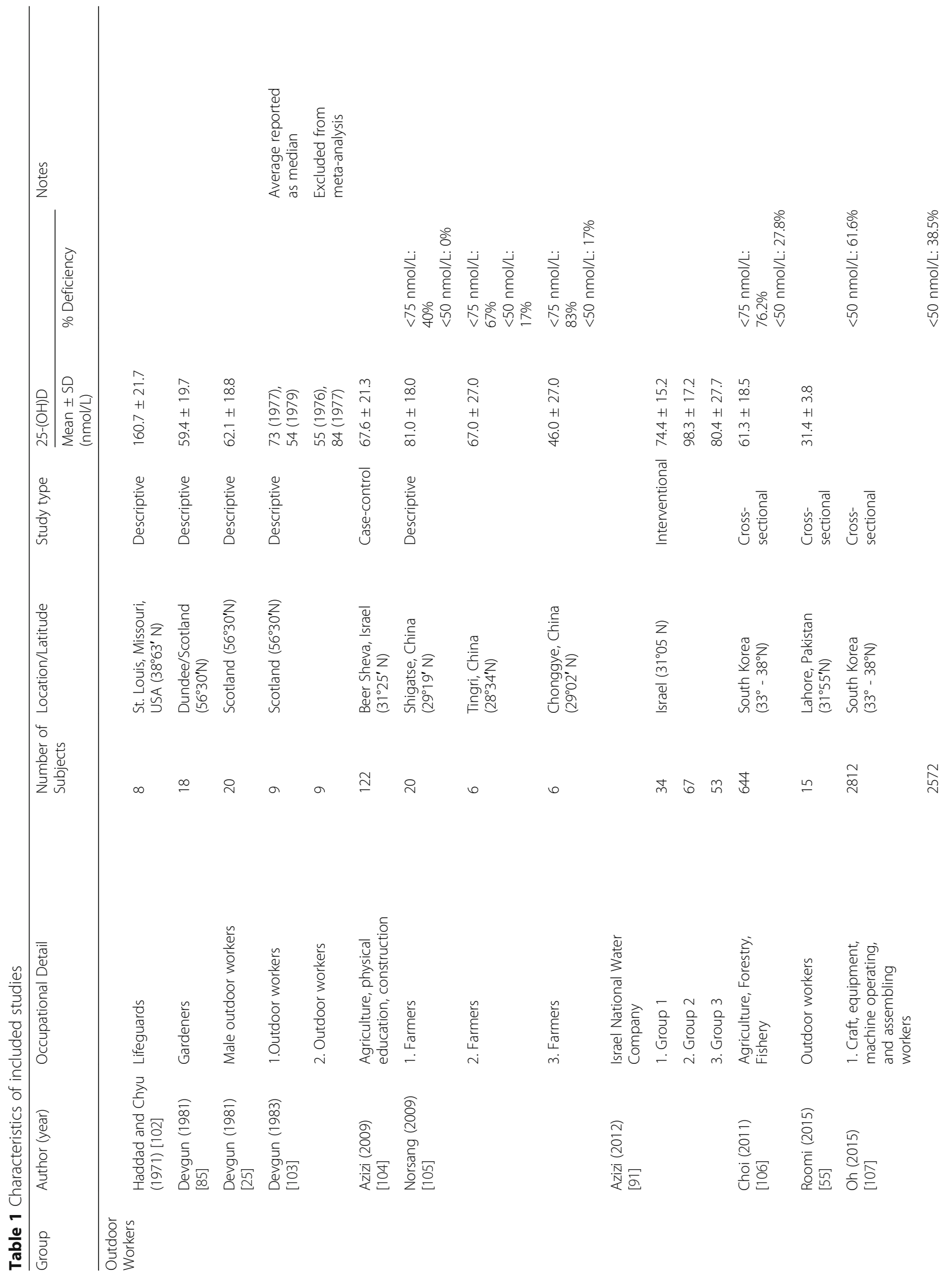


要

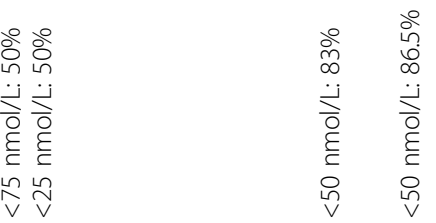

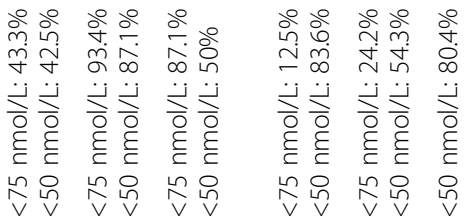

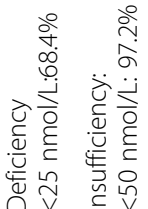

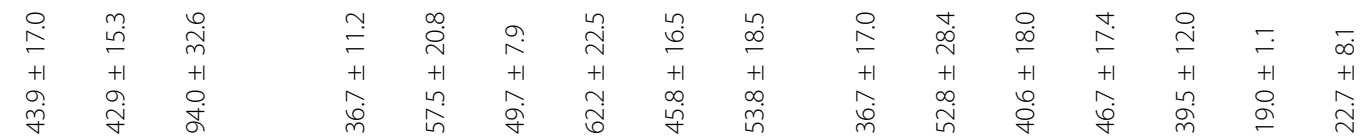

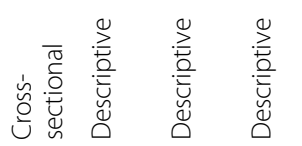

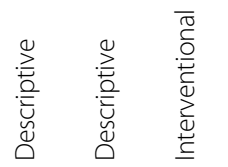

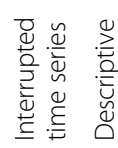

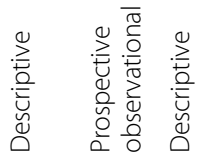

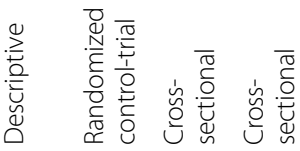

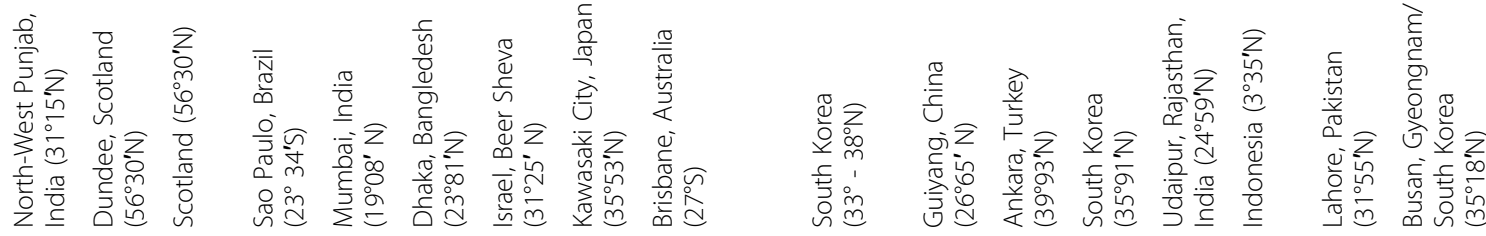

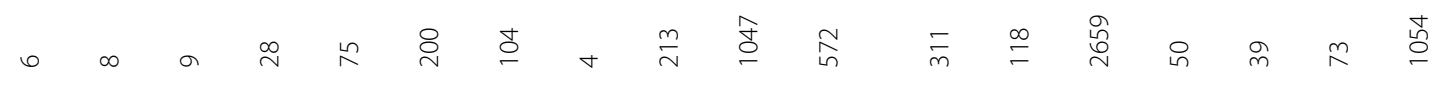

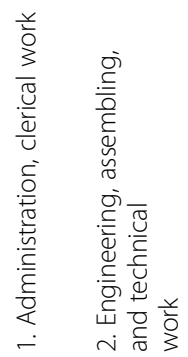

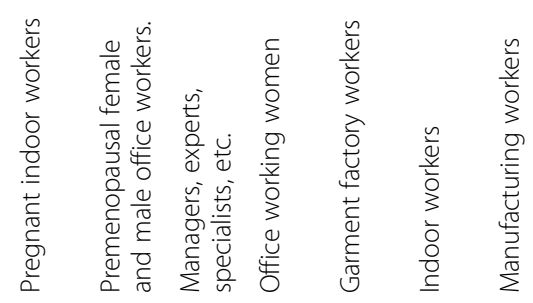

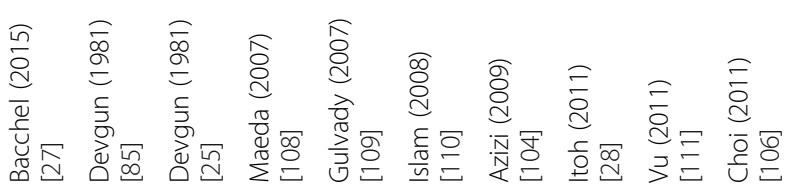

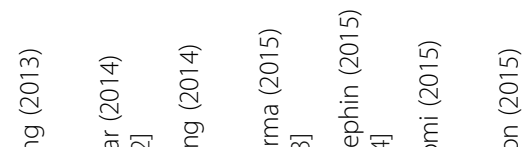

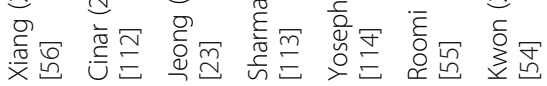

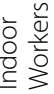




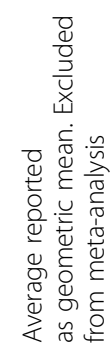

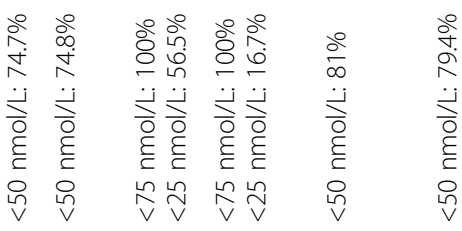
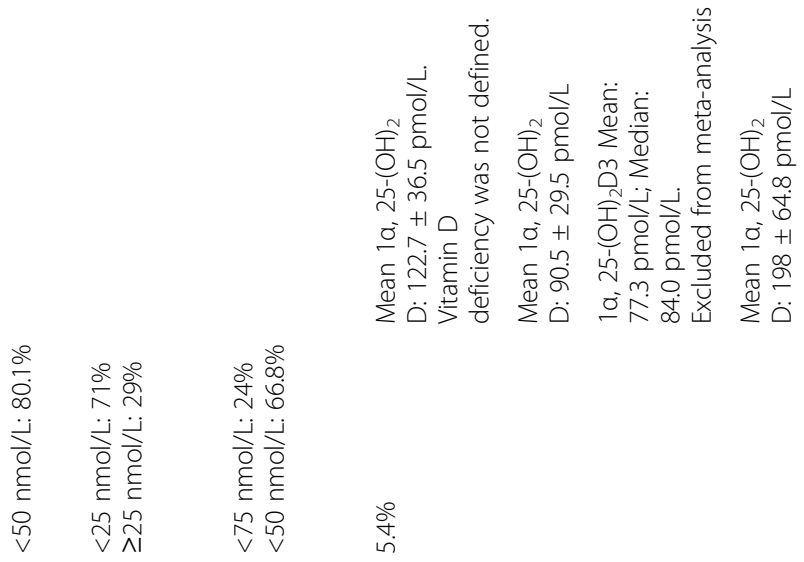

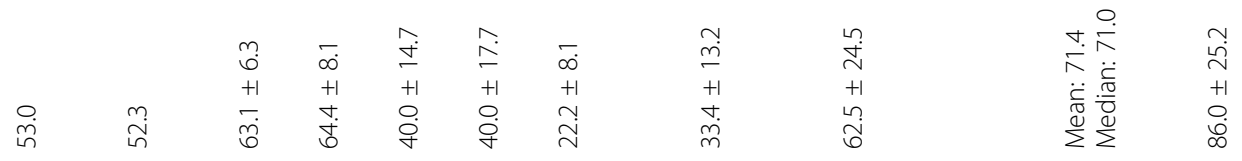

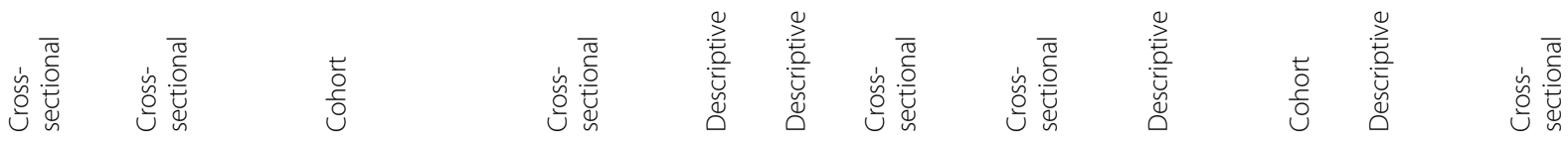

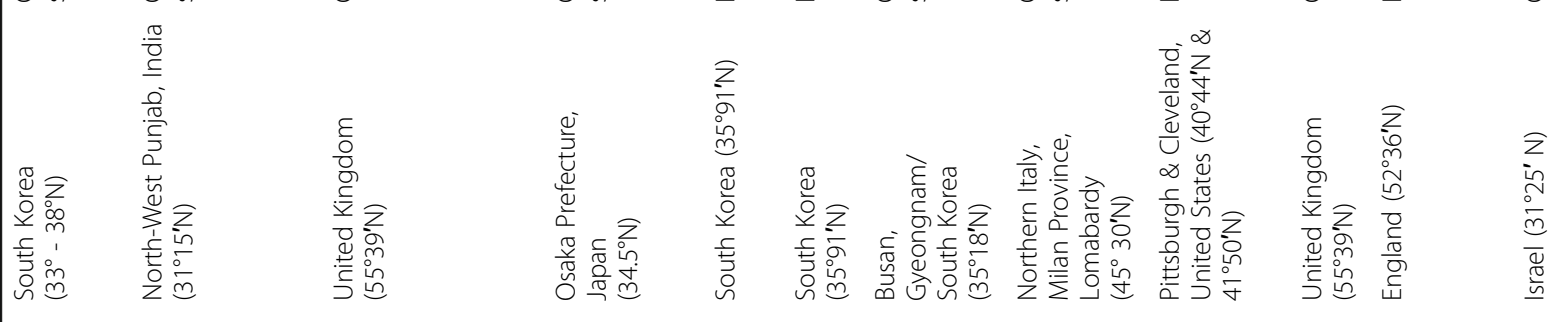

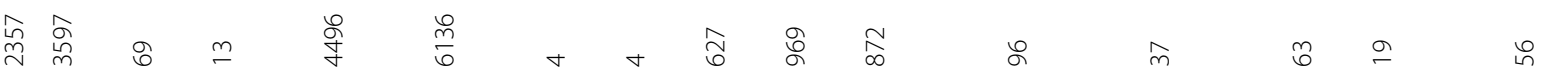
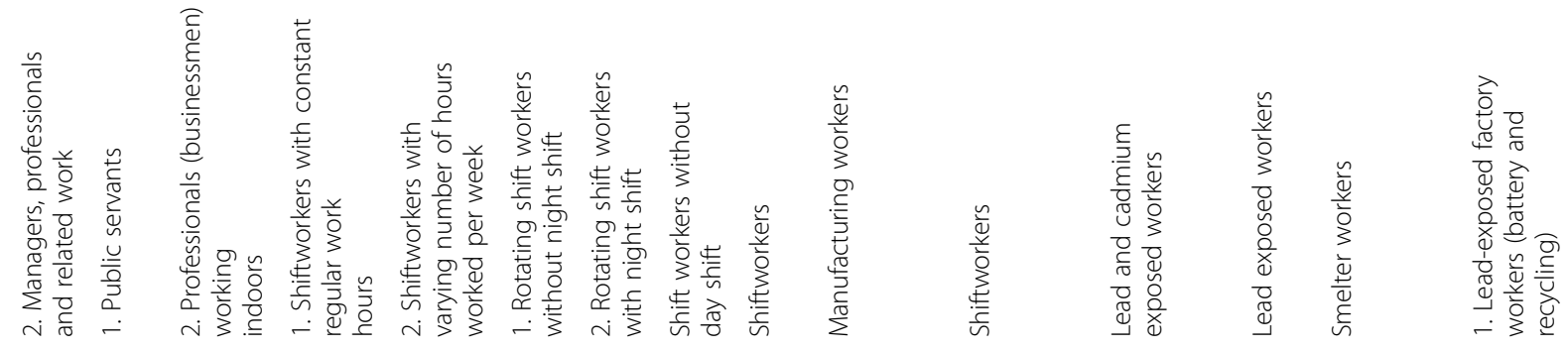

产

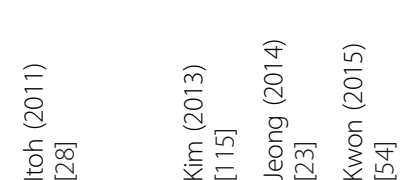

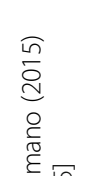

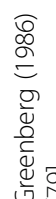

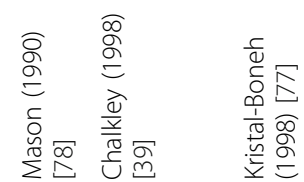
空产产

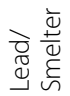




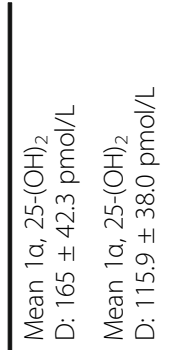

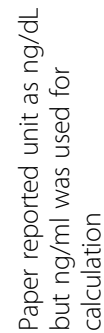

\section{ì}

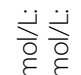

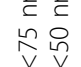

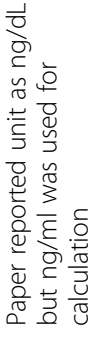

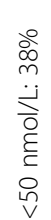

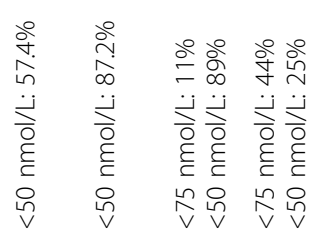

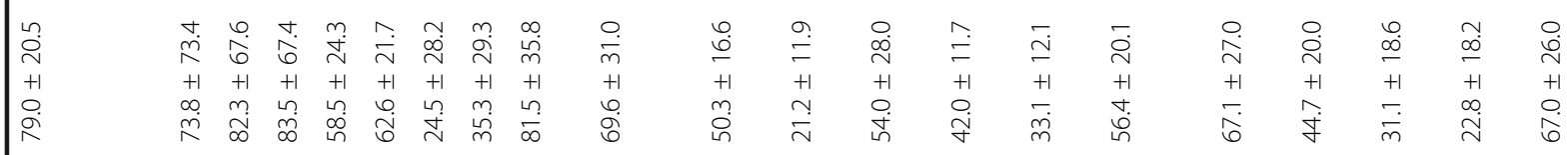

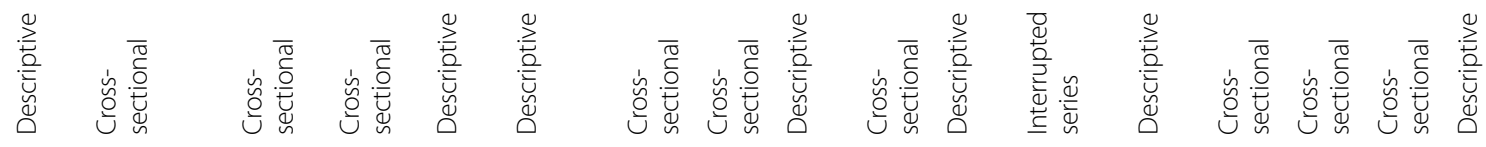

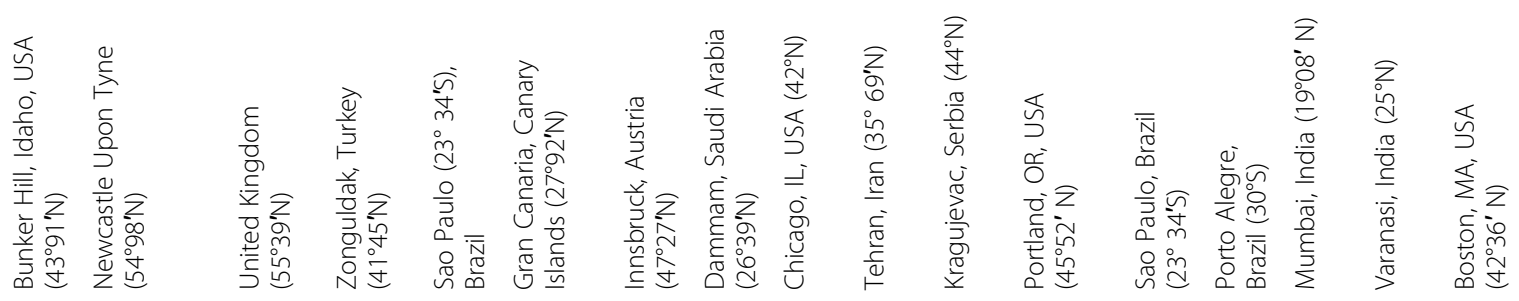

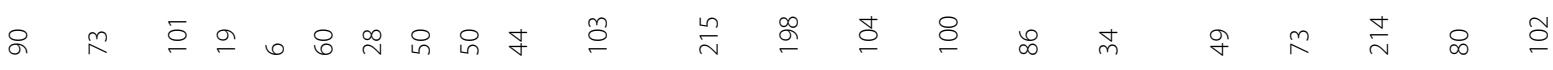




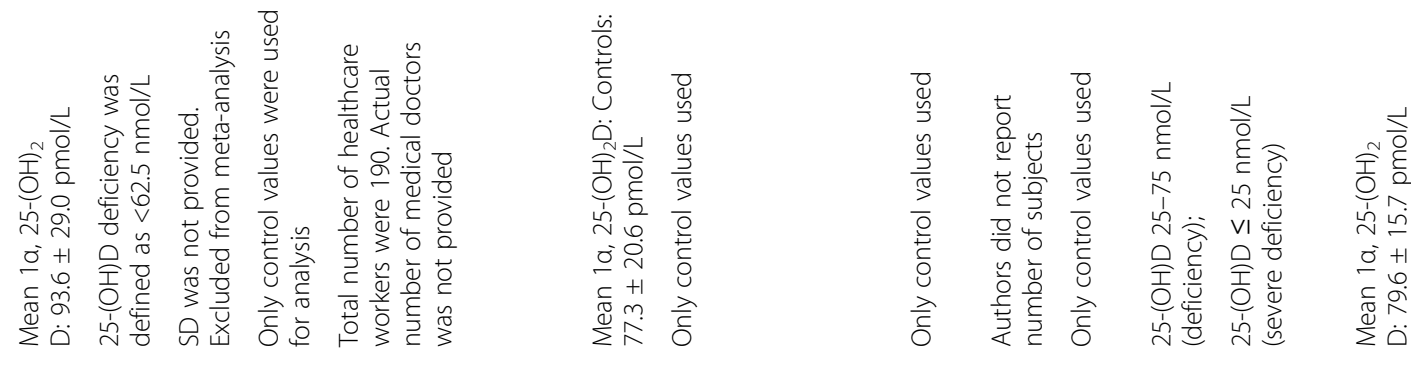

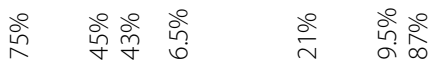

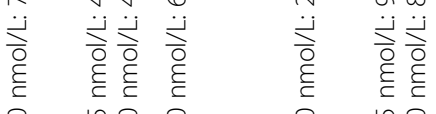

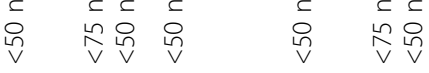
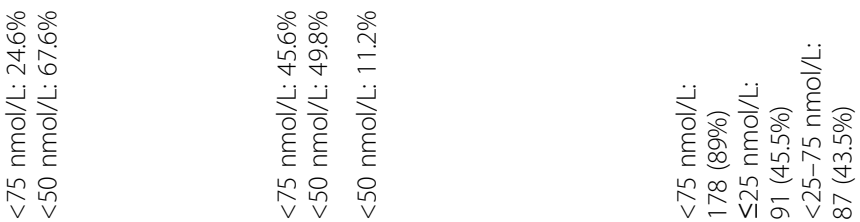

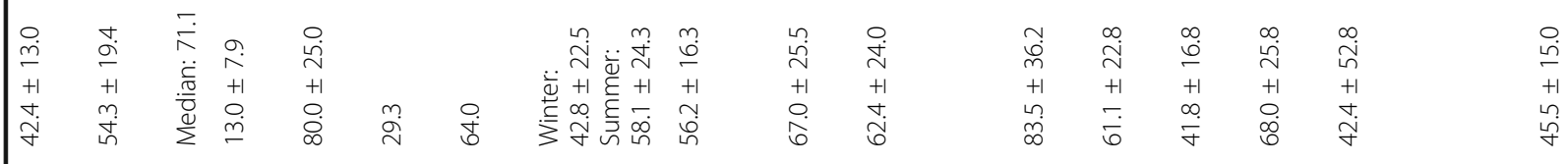

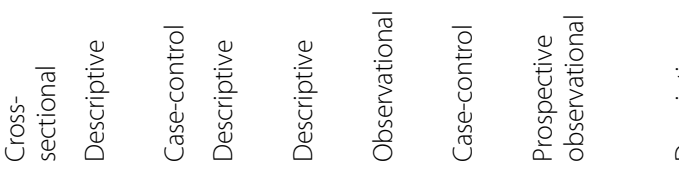

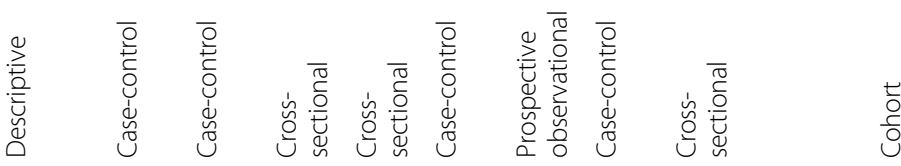

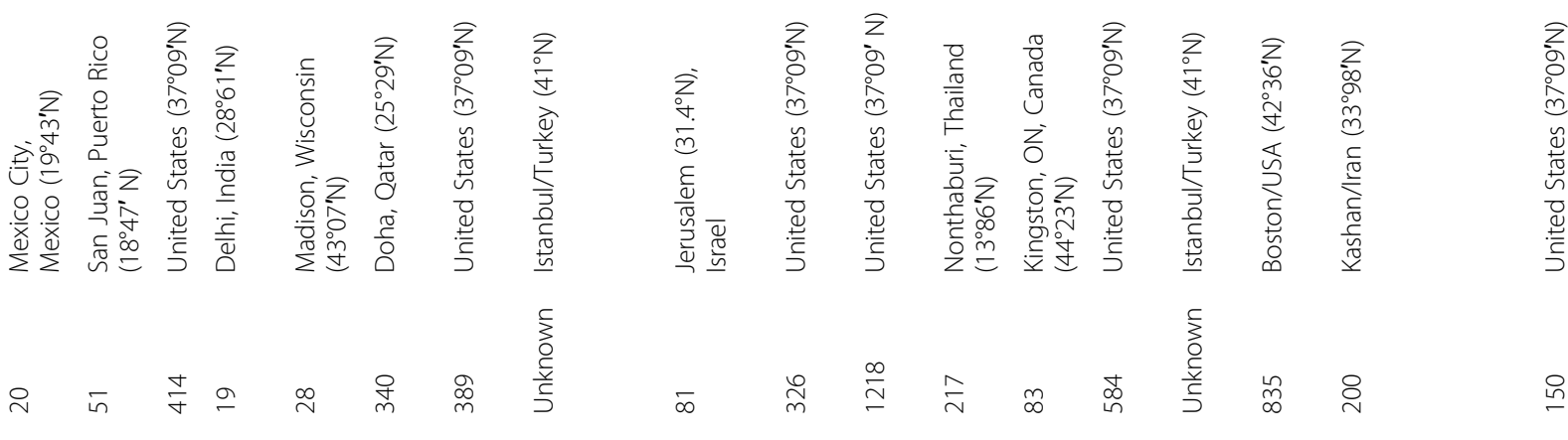


Sowah et al. BMC Public Health (2017) 17:519

Page 11 of 25

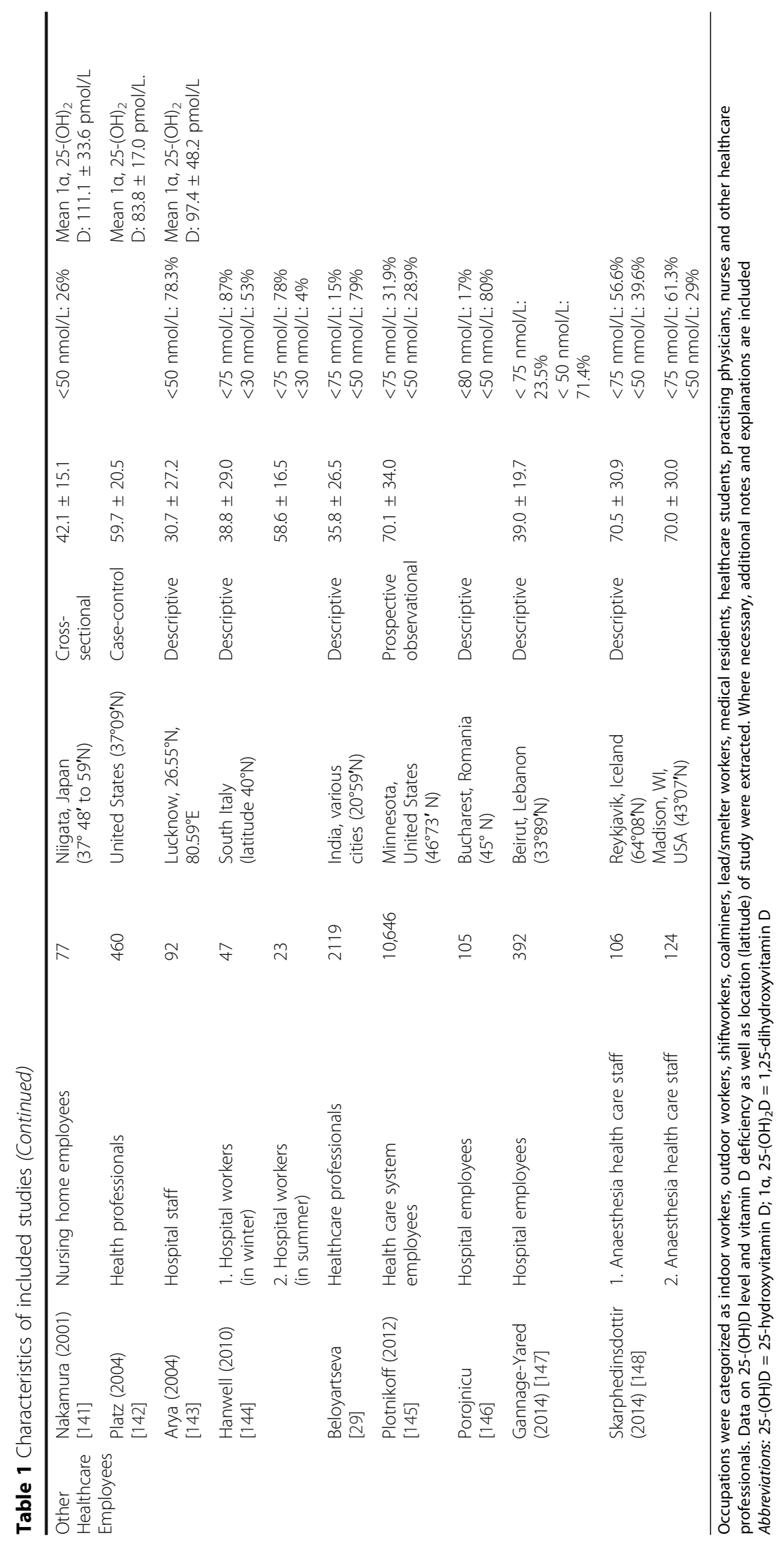


Table 2 Assay type, measure of coefficient of variation (reliability) and seasons of included studies

\begin{tabular}{|c|c|c|c|c|c|}
\hline Category & Study ID & Assay type & Reliability & Season of year & $\begin{array}{l}\text { Study } \\
\text { quality }\end{array}$ \\
\hline \multirow[t]{11}{*}{$\begin{array}{l}\text { Outdoor } \\
\text { Workers }\end{array}$} & $\begin{array}{l}\text { Haddad and Chyu } \\
\text { [102] }\end{array}$ & $\begin{array}{l}\text { Competitive protein } \\
\text { binding assay }\end{array}$ & CV: $8-14 \%$ & Spring, Summer & High \\
\hline & Devgun [85] & $\begin{array}{l}\text { Competitive protein } \\
\text { binding assay }\end{array}$ & Inter-assay CV: 10\% & All year & High \\
\hline & Devgun [25] & $\begin{array}{l}\text { Competitive protein } \\
\text { binding assay }\end{array}$ & Inter-assay CV: 10.7\% & All year & High \\
\hline & Devgun [103] & $\begin{array}{l}\text { Competitive protein binding } \\
\text { assay }\end{array}$ & Inter-assay CV: 10.6\% & Autumn, Winter & High \\
\hline & Azizi [104] & $\begin{array}{l}\text { Competitive protein } \\
\text { binding assay }\end{array}$ & Unknown & All year & Medium \\
\hline & Norsang [105] & RIA & Unknown & Autumn & Medium \\
\hline & Azizi [91] & RIA & Unknown & Winter & Medium \\
\hline & Choi [106] & RIA & Inter-assay CV: 11.7-12.5\% & All year & High \\
\hline & Roomi [55] & EIA & Inter-assay CV: 4.9\% & All year & High \\
\hline & Oh [107] & RIA & Unknown & Unknown & Low \\
\hline & Bacchelv [27] & Unknown & Unknown & Unknown & Low \\
\hline \multirow[t]{18}{*}{ Indoor Workers } & Devgun [85] & $\begin{array}{l}\text { Competitive protein } \\
\text { binding assay }\end{array}$ & Inter-assay CV: 10\% & All year & High \\
\hline & Devgun [25] & $\begin{array}{l}\text { Competitive protein } \\
\text { binding assay }\end{array}$ & Inter-assay CV: 10.7\% & All year & High \\
\hline & Maeda [108] & $\begin{array}{l}\text { Immunoradiometric } \\
\text { assay }\end{array}$ & $\begin{array}{l}\text { Inter-assay CV: } 16 \% \text { (for lowest values; } \\
3 \% \text { for highest values) } \\
\text { Intra-assay: } 4.8 \%\end{array}$ & $\begin{array}{l}\text { Summer, Spring, } \\
\text { Winter }\end{array}$ & High \\
\hline & Gulvady [109] & RIA & Unknown & Unknown & Low \\
\hline & Islam [110] & EIA & Inter-assay CV: 7\% Intra-assay CV: 5.4\% & Unknown & Medium \\
\hline & Azizi [104] & $\begin{array}{l}\text { Competitive protein } \\
\text { binding assay }\end{array}$ & Unknown & All year & Medium \\
\hline & Itoh [28] & RIA/EIA & Inter -assay CV: 21.9 & Winter, Autumn & High \\
\hline & Vu [111] & Chemiluminescent assay & $\begin{array}{l}\text { Inter-assay CV: 6-9\% } \\
\text { Intra-assay CV: 3-6\% }\end{array}$ & Summer, Winter & High \\
\hline & Choi [106] & RIA & Inter-assay CV: 11.7-12.5\% & All year & High \\
\hline & Xiang [56] & HPLC-MS/MS tandem & $\begin{array}{l}\text { Inter-assay CV: } 6.9-9.5 \% \\
\text { Intra-assay CV: } 2.77-3.2 \%\end{array}$ & All year & High \\
\hline & Cinar [112] & HPLC & $\begin{array}{l}\text { Inter-assay CV: } 3.4 \% \\
\text { Intra-assay: } 4.3 \%\end{array}$ & $\begin{array}{l}\text { Summer, } \\
\text { Winter }\end{array}$ & High \\
\hline & Jeong [23] & Unknown & Unknown & Unknown & Low \\
\hline & Sharma [113] & Chemiluminescent assay & Unknown & Unknown & Low \\
\hline & Yosephin [114] & EIA & Unknown & Unknown & Low \\
\hline & Roomi [55] & EIA & Inter-assay CV: $4.9 \%$ & All year & High \\
\hline & Kwon [54] & EIA & Unknown & Winter, Spring & Medium \\
\hline & Oh [107] & RIA & Unknown & Unknown & Low \\
\hline & Bacchel [27] & Unknown & Unknown & Unknown & Low \\
\hline \multirow[t]{4}{*}{ Shiftworkers } & Ward [41] & ELISA & $\begin{array}{l}\text { Intra-assay CV: } 5.5-7.2 \% \text { (concentrations } \\
\text { standardized according to mean of values } \\
\text { from vitamin D External Quality Assurance } \\
\text { Survey) }\end{array}$ & Unknown & Medium \\
\hline & Itoh [28] & RIA & Intra- and inter-day variation: $4.3-7 \%$ & Summer & High \\
\hline & Kim [115] & RIA & Unknown & All year & Medium \\
\hline & Jeong [23] & Unknown & Unknown & Unknown & Low \\
\hline
\end{tabular}


Table 2 Assay type, measure of coefficient of variation (reliability) and seasons of included studies (Continued)

\begin{tabular}{|c|c|c|c|c|c|}
\hline & Kwon [54] & EIA & Unknown & Winter, Spring & Medium \\
\hline & Romano [45] & Chemiluminescent assay & Unknown & Spring & Medium \\
\hline \multirow[t]{5}{*}{ Lead/Smelter } & Greenberg [79] & $\begin{array}{l}\text { Competitive protein } \\
\text { binding assay }\end{array}$ & Unknown & Unknown & Low \\
\hline & Mason [78] & Radioreceptor assay & $\begin{array}{l}\text { Inter-assay CV: } 11.5 \% \\
\text { Intra-assay CV: } 6.9 \%\end{array}$ & Unknown & Medium \\
\hline & Chalkley [39] & RIA & Unknown & Unknown & Low \\
\hline & Kristal-Boneh [77] & $\begin{array}{l}\text { Competitive protein } \\
\text { binding assay }\end{array}$ & $\begin{array}{l}\text { Inter-assay CV: } 15.2 \% \\
\text { Intra-assay CV: } 3.9 \%\end{array}$ & Summer & High \\
\hline & Potula [116] & RIA & Unknown & Unknown & Low \\
\hline \multirow[t]{3}{*}{ Coalminers } & Shuster [82] & $\begin{array}{l}\text { Competitive protein } \\
\text { binding assay }\end{array}$ & Unknown & Spring, Summer & Medium \\
\hline & Shuster [81] & $\begin{array}{l}\text { Competitive protein } \\
\text { binding assay }\end{array}$ & Unknown & Winter, Autumn & Medium \\
\hline & Sarikaya [83] & ELISA & Unknown & Unknown & Low \\
\hline \multirow[t]{7}{*}{$\begin{array}{l}\text { Healthcare } \\
\text { Students }\end{array}$} & Maeda [108] & $\begin{array}{l}\text { Immunoradiometric } \\
\text { assay }\end{array}$ & $\begin{array}{l}\text { Inter-assay CV: } 16 \% \text { (for lowest values; } \\
3 \% \text { for highest values) } \\
\text { Intra-assay CV: } 4.8 \%\end{array}$ & $\begin{array}{l}\text { Summer, Spring, } \\
\text { Winter }\end{array}$ & High \\
\hline & $\begin{array}{l}\text { Gonzalez-Padilla } \\
\text { [117] }\end{array}$ & Immunochemiluminescence & $\begin{array}{l}\text { Inter-assay CV: } 7.1-10 \% \\
\text { Intra-assay CV: } 3-4.5 \%\end{array}$ & Unknown & Medium \\
\hline & Kaehler [118] & Electro-chemoluminescence & Unknown & Spring & Medium \\
\hline & Al-Elq [119] & Chemiluminescent assay & Unknown & Winter & Medium \\
\hline & Manickam [120] & Chemiluminescent assay & $\begin{array}{l}\text { Inter-assay CV: } 13.9 \% \\
\text { Intra-assay CV: } 10.8 \%\end{array}$ & Unknown & Medium \\
\hline & $\begin{array}{l}\text { Zabihiyeganeh } \\
{[121]}\end{array}$ & RIA & $\begin{array}{l}\text { Inter-assay CV: 6.4\% } \\
\text { Intra-assay CV: } 5.6 \%\end{array}$ & Autumn & High \\
\hline & Milovanovic [122] & Unknown & Unknown & Spring, Summer & Low \\
\hline \multirow{8}{*}{$\begin{array}{l}\text { Medical } \\
\text { Residents }\end{array}$} & Haney [61] & RIA & Unknown & Autumn, Spring & Medium \\
\hline & Maeda [108] & Immunoradiometric assay & $\begin{array}{l}\text { Inter-assay CV: } 16 \% \text { (for lowest values; } \\
3 \% \text { for highest values) } \\
\text { Intra-assay: } 4.8 \%\end{array}$ & $\begin{array}{l}\text { Summer, Spring, } \\
\text { Winter }\end{array}$ & High \\
\hline & Premaor [123] & Chemiluminescent assay & Intra-assay CV: 6\% & Autumn & High \\
\hline & Multani [76] & RIA & $\begin{array}{l}\text { Inter-assay CV: 6.49\% } \\
\text { Intra-assay CV: } 3.85 \%\end{array}$ & Spring, Summer & High \\
\hline & Singh [124] & RIA & Unknown & Winter, Summer & Medium \\
\hline & Growdon [125] & RIA & $\begin{array}{l}\text { Inter-assay CV: } 6.2-12.5 \% \\
\text { Intra-assay CV: } 4.4-8.3 \%\end{array}$ & Winter & High \\
\hline & Mendoza [126] & Chemiluminescent assay & $\begin{array}{l}\text { Inter-assay CV: } 2.1 \% \\
\text { Intra-assay CV: } 4.1 \%\end{array}$ & Summer & High \\
\hline & Ramirez-Vick [127] & LC-MS/MS & Unknown & Spring, Winter & Medium \\
\hline \multirow{7}{*}{$\begin{array}{l}\text { Practising } \\
\text { Physicians }\end{array}$} & Gann [128] & RIA & Intra-assay CV: 8.1\% & Unknown & Medium \\
\hline & Goswami [129] & RIA & $\begin{array}{l}\text { Inter-assay CV: } 13 \% \\
\text { Intra-assay CV: } 8 \%\end{array}$ & Winter, Summer & High \\
\hline & Kramm [130] & HPLC & Unknown & Unknown & Low \\
\hline & Mahdy [75] & Unknown & Unknown & Unknown & Low \\
\hline & Lee [131] & RIA & Intra-assay CV: 13.8\% & Summer, Autumn & high \\
\hline & Haliloglu [132] & HPLC & $\begin{array}{l}\text { Inter-assay CV: } 3.1-4.7 \% \\
\text { Intra-assay CV: } 0.7-4.9 \%\end{array}$ & Winter, Summer & High \\
\hline & Munter [133] & Chemiluminescent assay & Unknown & Unknown & Low \\
\hline \multirow[t]{2}{*}{ Nurses } & Platz [134] & RIA & Intra-assay CV: 7.5\% & Unknown & Medium \\
\hline & Eliassen [135] & RIA & Overall CV: $10.7 \%$ and $6 \%$ & Unknown & Medium \\
\hline
\end{tabular}


Table 2 Assay type, measure of coefficient of variation (reliability) and seasons of included studies (Continued)

\begin{tabular}{|c|c|c|c|c|c|}
\hline & $\begin{array}{l}\text { Hattapornsawan } \\
\text { [136] }\end{array}$ & LC-MS/MS & $\begin{array}{l}\text { Inter-assay CV: } 6.3 \% \\
\text { Intra-assay CV: } 5 \%\end{array}$ & Unknown & Medium \\
\hline & Wallingford [137] & RIA & Intra-assay CV: 0.99\% & All year & High \\
\hline & Wang [138] & RIA & Overall CV: $10.7 \%$ and $6 \%$ & Unknown & Medium \\
\hline & Haliloglu [132] & HPLC & $\begin{array}{l}\text { Inter-assay CV: } 3.1-4.7 \% \\
\text { Intra-assay CV: } 0.7-4.9 \%\end{array}$ & Winter, Summer & High \\
\hline & Bertrand [139] & $\begin{array}{l}\text { High affinity protein-binding } \\
\text { assay or RIA }\end{array}$ & Overall CV: $17.6 \%$ and $6 \%$ & Unknown & Medium \\
\hline & Madani [140] & ELISA & Unknown & Summer & Medium \\
\hline \multirow{11}{*}{$\begin{array}{l}\text { Other Healthcare } \\
\text { Professionals }\end{array}$} & Platz [59] & RIA & Intra-assay CV: 6.7\% & Unknown & Medium \\
\hline & Nakamura [141] & HPLC & Inter-assay CV: 2.6-4.2\% & Winter & High \\
\hline & Platz [142] & RIA & Intra-assay CV: $5.4-5.6 \%$ & All year & High \\
\hline & Arya [143] & RIA & Inter-assay CV: 8.4\% & Unknown & Medium \\
\hline & Hanwell [144] & RIA & $\begin{array}{l}\text { Inter-assay CV: 12\% } \\
\text { Intra-assay CV: } 7.2 \%\end{array}$ & Winter, Summer & High \\
\hline & Beloyartseva [29] & RIA & Unknown & Winter & Medium \\
\hline & Plotnikoff [145] & Chemiluminescent assay & CV: $9.8-12.5 \%$ & Winter, Spring & High \\
\hline & Porojnicu [146] & HPLC & Inter-assay CV: 12\% & Winter & High \\
\hline & $\begin{array}{l}\text { Gannage-Yared } \\
\text { [147] }\end{array}$ & Chemiluminescent assay & Inter- and Intra CV: $<12 \%$ & All year & High \\
\hline & $\begin{array}{l}\text { Skarphedinsdottir } \\
{[148]}\end{array}$ & HPLC & Unknown & Spring & Medium \\
\hline & Haliloglu [132] & HPLC & $\begin{array}{l}\text { Inter-assay CV: } 3.1-4.7 \% \\
\text { Intra-assay CV: } 0.7-4.9 \%\end{array}$ & Winter, Summer & High \\
\hline
\end{tabular}

The assay type, coefficient of variation and the season of each study were extracted to assess the methodological quality of each study

Abbreviations: CV coefficient of variation, RIA radioimmunoassay, LC-MS/MS liquid chromatography-mass spectrometry/mass spectrometry, ELISA enzyme-linked immunosorbent assay, EIA electrochemiluminescence immunoassay, HPLC high performance liquid chromatography

insufficiency in addition to deficiency. In this one study, which was conducted at latitude $45^{`} 30^{\circ} \mathrm{N}$, about $91 \%$ of subjects were found to be vitamin $\mathrm{D}$ deficient or insufficient [45] (Fig. 2). As shown in Tables 3 and 4, shiftworkers had the highest risk to develop vitamin D deficiency (RR: $1.27 ; 95 \%$ CI: 1.26 to 1.28$)$ and a RR of $1.24(95 \%$ CI: 1.16 to 1.32 ) to develop vitamin D insufficiency.

\section{Lead and smelter workers}

Five studies of 183 subjects reported on lead and smelter workers. The mean vitamin D level of lead/ smelter workers was $77.8 \pm 5.4 \mathrm{nmol} / \mathrm{L}$; they represented the occupational group with the highest vitamin D level among all the occupational categories we investigated (Fig. 1). None of the studies presented data on the proportion of subjects who were either vitamin $\mathrm{D}$ deficient or insufficient. All the included studies on lead/smelter workers also measured circulating levels of $1 \alpha, 25-(\mathrm{OH})_{2} \mathrm{D}$, the active metabolite of vitamin $\mathrm{D}$. In a sub-analysis, we showed that the average level of $1 \alpha, 25-(\mathrm{OH})_{2} \mathrm{D}$ in lead/smelter workers was $139.73 \pm 57.51$ (mean \pm SD) $\mathrm{pmol} / \mathrm{L}$ (Table 1).

\section{Coalminers}

There was a paucity of studies that investigated the status of vitamin D in coalminers in the literature. Only 3 studies assessed vitamin D levels in coalminers, with an overall total of 314 subjects. The average serum vitamin D level in coalminers was $56.6 \pm 28.4 \mathrm{nmol} / \mathrm{L}$ (Table 1 ). In order to evaluate the impact of the type of mining on vitamin D status, we further divided coalminers into underground and surface miners. Our analysis revealed that there was no statistically significant difference in average vitamin D levels between underground and surface miners $(57.8 \pm 11.7$ vs. $52.4 \pm 12.4 \mathrm{nmol} / \mathrm{L}$, $p=0.78$ ) (Additional file 7: Figure S2). None of the three studies analyzed reported data on the number of subjects that were vitamin $\mathrm{D}$ deficient or insufficient.

\section{Healthcare workers}

We found that the overall mean serum 25-(OH)D level of all healthcare workers was $61.6 \pm 11.0 \mathrm{nmol} / \mathrm{L}$ (data from 19,083 study subjects from 35 different studies). Among healthcare workers, our analysis demonstrated that medical residents and healthcare students have the lowest level of circulating vitamin $\mathrm{D}(44.0 \pm 8.3 \mathrm{nmol} / \mathrm{L}$ and $45.2 \pm 5.5 \mathrm{nmol} / \mathrm{L}$, respectively) and there was no 


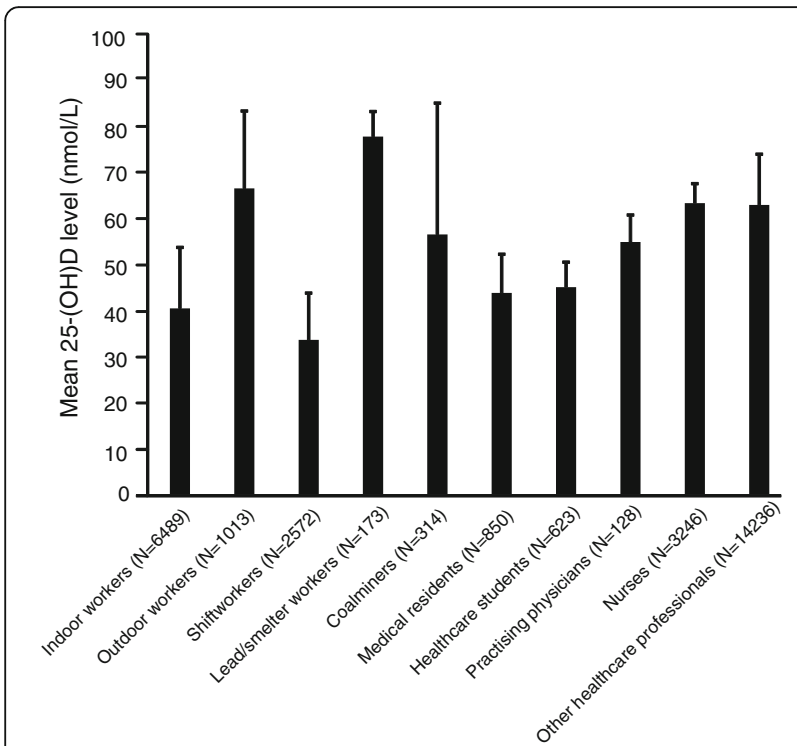

Fig. 1 25-hydroxyvitamin D (25-(OH)D) levels in different occupational groups. Data represent the weighted means pooled from the means of the included studies obtained for each occupational category. Error bars represent pooled standard error of means computed as SEP $=S p \sqrt{\frac{1}{n_{1}}+\frac{1}{n_{2}}}$, where Sp is pooled variance, $n_{1}$ represents sample size of group 1 , and $n_{2}$ represents sample size of group 2

statistically significant difference $(p=0.9)$ between these two sub-groups (Additional file 8: Figure S3). Additionally, $65 \%$ and $72 \%$ of medical residents and healthcare students, respectively, were vitamin D deficient (Fig. 2). According to Tables 3 and 4, medical residents had RR of 1.04 (95\% CI: 0.97 to 1.10) of vitamin D deficiency and the RR was 1.14 for healthcare students (95\% CI: 1.09 to 1.19). With respect to vitamin D insufficiency, medical residents had a RR of 1.20 (95\% CI: 1.15 to 1.26 ) and the RR was 1.20 (95\% CI: 1.16 to 1.23 ) for healthcare students.

Seven studies reported on vitamin D in practising physicians, but only three studies comprising 128 subjects reported 25- $(\mathrm{OH}) \mathrm{D}$ levels; the mean $25-(\mathrm{OH}) \mathrm{D}$ level was $55.0 \pm 5.8 \mathrm{nmol} / \mathrm{L}$ (Table 1 and Additional file 8: Figure S3). Relative to medical residents and healthcare students, the higher level of 25- $(\mathrm{OH}) \mathrm{D}$ in practising physicians was statistically significant $(p<0.001$ for the comparison against each group). Vitamin D deficiency in practising physicians, reported in four studies (835 subjects) was 46\%, significantly lower than that seen in medical residents and healthcare students $(p<0.001$ and $p<0.001$, respectively) (Fig. 2). As shown in Tables 3 and 4, practising physicians had a RR of 0.73 (95\% CI: 0.68 to 0.78 ) and 1.30 (95\% CI: 1.28 to 1.33 ) of vitamin D deficiency and insufficiency, respectively.

The average serum 25- $(\mathrm{OH}) \mathrm{D}$ level in 3246 nurses from 8 studies was $63.4 \pm 4.2 \mathrm{nmol} / \mathrm{L}$ (Fig. 1 and Additional file 8: Figure S3). The difference between

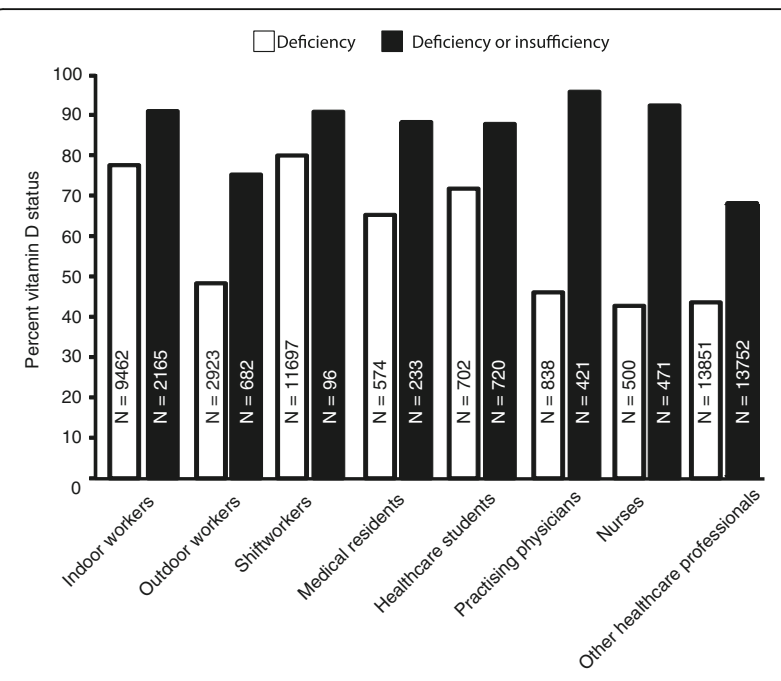

Fig. 2 Percent vitamin D status in different occupational groups. Vitamin D deficiency (white bars) was defined according to the Endocrine Society's (ES) categorization as a serum level of 25$(\mathrm{OH}) \mathrm{D} \leq 50 \mathrm{nmol} / \mathrm{L}(20 \mathrm{ng} / \mathrm{ml})$. Each white bar graph represents \% of subjects of each group with a serum $25-(\mathrm{OH}) \mathrm{D} \leq 50 \mathrm{nmol} / \mathrm{L}$. The black bars represent percent vitamin $D$ deficiency or insufficiency in different occupational groups. Vitamin D insufficiency was defined based on the ES's criteria, which indicates a serum level of 25$(\mathrm{OH}) \mathrm{D} \leq 75 \mathrm{nmol} / \mathrm{L}(30 \mathrm{ng} / \mathrm{ml})$ as insufficient. Each black bar graph represents the \% of subjects of each group with a serum $25-(\mathrm{OH}) \mathrm{D}$ level $\leq 75 \mathrm{nmol} / \mathrm{L}$. The numbers within the bars, $\mathrm{N}$, represent the total number of subjects contributing to each category for whom vitamin D deficiency, insufficiency, or sufficiency could be determined

mean vitamin D status in nurses compared to medical residents, healthcare students or practising physicians, was statistically significant $(p<0.0001$ vs. both medical students and healthcare students; $p<0.01$ vs. practising physicians; Fig. 1). Furthermore, our analysis showed that $43 \%$ of 500 nurses were deficient in serum 25$(\mathrm{OH}) \mathrm{D}$ (Fig 2). The proportion of nurses deficient in vitamin $\mathrm{D}$ was not significantly different compared to practising physicians $(p=0.6)$, but differed significantly when compared with medical residents and healthcare students ( $p<0.001$ and $p=0.02$, respectively; Fig. 2). Nurses had a RR of 0.68 (95\% CI: 0.61 to 0.75 ) to develop vitamin D deficiency and a RR of 1.26 (95\% CI: 1.22 to 1.29 ) of insufficiency (Tables 3 and 4).

The final sub-division of the healthcare category was the group of employees we termed 'other healthcare employees', which comprised all employees in healthcare who were not specifically identified as nurses, physicians, medical residents or healthcare students. Eleven studies of 14,236 subjects reported on vitamin D level in this group of workers and the average vitamin $\mathrm{D}$ level was $63.0 \pm 11.0 \mathrm{nmol} / \mathrm{L}$, similar to values obtained in nurses, as described above. Similar to nurses, $43 \%$ of the other healthcare employees group were vitamin D deficient (Fig 
Table 3 Occupational groups, \% deficiency, and relative risk

\begin{tabular}{|c|c|c|c|c|}
\hline $\begin{array}{l}\text { Occupational } \\
\text { group }\end{array}$ & $\begin{array}{l}\text { Number of } \\
\text { subjects }\end{array}$ & $\begin{array}{l}\text { Number of } \\
\text { vitamin D } \\
\text { deficient } \\
\text { subjects }\end{array}$ & $\begin{array}{l}\text { \% deficiency } \\
(25-(\mathrm{OH}) \mathrm{D} \\
<50 \mathrm{nmol} / \mathrm{L})\end{array}$ & Relative risk \\
\hline $\begin{array}{l}\text { All groups } \\
\text { (total) }\end{array}$ & 46,426 & 29,255 & 63.0 & 1.00 (baseline) \\
\hline $\begin{array}{l}\text { Indoor } \\
\text { workers }\end{array}$ & 12,204 & 9462 & 77.5 & $\begin{array}{l}1.23(95 \% \mathrm{Cl}: 1.22 \\
\text { to } 1.24)\end{array}$ \\
\hline $\begin{array}{l}\text { Outdoor } \\
\text { workers }\end{array}$ & 6060 & 2923 & 48.2 & $\begin{array}{l}0.77 \text { (95\% Cl: } 0.75 \\
\text { to } 0.79 \text { ) }\end{array}$ \\
\hline Shiftworkers & 11,697 & 9354 & 80 & $\begin{array}{l}1.27(95 \% \mathrm{Cl}: 1.26 \\
\text { to } 1.28)\end{array}$ \\
\hline $\begin{array}{l}\text { Medical } \\
\text { residents }\end{array}$ & 574 & 375 & 65.3 & $\begin{array}{l}1.04(95 \% \mathrm{Cl}: 0.97 \\
\text { to } 1.10)\end{array}$ \\
\hline $\begin{array}{l}\text { Healthcare } \\
\text { students }\end{array}$ & 702 & 504 & 71.6 & $\begin{array}{l}1.14(95 \% \mathrm{Cl}: 1.09 \\
\text { to } 1.19)\end{array}$ \\
\hline $\begin{array}{l}\text { Practising } \\
\text { physicians }\end{array}$ & 838 & 386 & 46.1 & $\begin{array}{l}0.73(95 \% \mathrm{Cl}: 0.68 \\
\text { to } 0.78)\end{array}$ \\
\hline Nurses & 500 & 213 & 42.3 & $\begin{array}{l}068(95 \% \mathrm{Cl}: 0.61 \\
\text { to } 0.75)\end{array}$ \\
\hline $\begin{array}{l}\text { Other } \\
\text { healthcare } \\
\text { workers }\end{array}$ & 13,851 & 6038 & 43.6 & $\begin{array}{l}0.69(95 \% \mathrm{Cl}: 0.68 \\
\text { to } 0.71)\end{array}$ \\
\hline
\end{tabular}

Vitamin $D$ deficiency was defined as $25-(\mathrm{OH}) \mathrm{D}<50 \mathrm{nmol} / \mathrm{L}$ Abbreviation: $\mathrm{Cl}$ confidence interval

Table 4 Occupational groups, combined \% insufficiency and deficiency, and relative risk

\begin{tabular}{|c|c|c|c|c|}
\hline $\begin{array}{l}\text { Occupational } \\
\text { group }\end{array}$ & $\begin{array}{l}\text { Number } \\
\text { of } \\
\text { subjects }\end{array}$ & $\begin{array}{l}\text { Number of } \\
\text { vitamin D } \\
\text { insufficient } \\
\text { subjects }\end{array}$ & $\begin{array}{l}\% \text { insufficiency } \\
(25-(\mathrm{OH}) \mathrm{D} \\
<75 \mathrm{nmol} / \mathrm{L})\end{array}$ & Relative risk \\
\hline $\begin{array}{l}\text { All groups } \\
\text { (total) }\end{array}$ & 18,704 & 13,735 & 73.4 & 1.00 (baseline) \\
\hline $\begin{array}{l}\text { Indoor } \\
\text { workers }\end{array}$ & 2383 & 2165 & 90.9 & $\begin{array}{l}1.24(95 \% \mathrm{Cl}: 1.22 \\
\text { to } 1.25)\end{array}$ \\
\hline $\begin{array}{l}\text { Outdoor } \\
\text { workers }\end{array}$ & 682 & 513 & 75.2 & $\begin{array}{l}1.02(95 \% \mathrm{Cl}: 0.98 \\
\text { to } 1.07)\end{array}$ \\
\hline Shiftworkers & 96 & 91 & 90.8 & $\begin{array}{l}1.24(95 \% \mathrm{Cl}: 1.16 \\
\text { to } 1.32)\end{array}$ \\
\hline $\begin{array}{l}\text { Medical } \\
\text { residents }\end{array}$ & 233 & 205 & 88.2 & $\begin{array}{l}1.20(95 \% \mathrm{Cl}: 1.15 \\
\text { to } 1.26)\end{array}$ \\
\hline $\begin{array}{l}\text { Healthcare } \\
\text { students }\end{array}$ & 720 & 632 & 87.8 & $\begin{array}{l}1.20(95 \% \mathrm{Cl}: 1.16 \\
\text { to } 1.23)\end{array}$ \\
\hline $\begin{array}{l}\text { Practising } \\
\text { physicians }\end{array}$ & 421 & 403 & 95.7 & $\begin{array}{l}1.30(95 \% \mathrm{Cl}: 1.28 \\
\text { to } 1.33)\end{array}$ \\
\hline Nurses & 417 & 385 & 92.3 & $\begin{array}{l}1.26(95 \% \mathrm{Cl}: 1.22 \\
\text { to } 1.29)\end{array}$ \\
\hline $\begin{array}{l}\text { Other } \\
\text { healthcare } \\
\text { workers }\end{array}$ & 13,752 & 9341 & 67.9 & $\begin{array}{l}0.93(95 \% \text { Cl: } 0.91 \\
\text { to } 0.94)\end{array}$ \\
\hline
\end{tabular}

Vitamin D insufficiency was defined as $25-(\mathrm{OH}) \mathrm{D}<75 \mathrm{nmol} / \mathrm{L}$ Abbreviation: $\mathrm{Cl}$ confidence interval
2). Other healthcare workers had a RR of 0.69 (95\% CI: 0.68 to 0.71 ) of vitamin $\mathrm{D}$ deficiency and a RR of 0.93 (95\% CI: 0.91 to 0.94) for vitamin D insufficiency (Tables 3 and 4). The vitamin D level and proportion with deficiency in the 'other healthcare employees' differed significantly when compared with either medical residents $(p<0.0001)$, healthcare students $(p<0.0001)$ or practising physicians $(p<0.001)$, but not nurses $(p=0.9)$. Geographical latitude did not affect vitamin D levels and prevalence of deficiency or insufficiency among healthcare professionals (Additional file 3: Figure S4C; Additional file 5: Figure S5C; Additional file 6: Figure S6C).

Most of the studies did not provide data on the season of the year in which the studies were conducted; thus, we could not perform a quantitative analysis on the seasonal effect on vitamin D levels across the various occupational categories. However, enough studies on indoor and outdoor workers reported on the seasonal effect on vitamin D levels to enable us to conduct quantitative comparisons between these two groups. As shown in Fig. 3, regardless of the season, the mean level of 25$(\mathrm{OH}) \mathrm{D}$ in outdoor workers was different compared to indoor workers. Among outdoor workers the level of vitamin $\mathrm{D}$ was lowest in the spring $(57.7 \pm 6.2 \mathrm{nmol} / \mathrm{L})$ compared to the winter $(74.6 \pm 9.0 \mathrm{nmol} / \mathrm{L})$, the summer $(70.5 \pm 6.9 \mathrm{nmol} / \mathrm{L})$ and the autumn $(72.6 \pm 13.3 \mathrm{nmol} /$ $\mathrm{L})$. Additionally, the $25-(\mathrm{OH}) \mathrm{D}$ levels in summer and autumn in outdoor workers were significantly different from levels found in winter $(p<0.0001)$ and spring $(p<0.0001)$. Surprisingly, values in the winter were comparable to the summer and autumn values in outdoor workers. In indoor workers, the highest value of vitamin $\mathrm{D}$ was observed in the summer $(65.8 \pm 10.3 \mathrm{nmol} / \mathrm{L})$ and the lowest in the spring $(41.8 \pm 7.4 \mathrm{nmol} / \mathrm{L})$ and winter $(44.3 \pm 11.6 \mathrm{nmol} / \mathrm{L})$. When compared to autumn values $(53.5 \pm 11.8 \mathrm{nmol} / \mathrm{L})$,

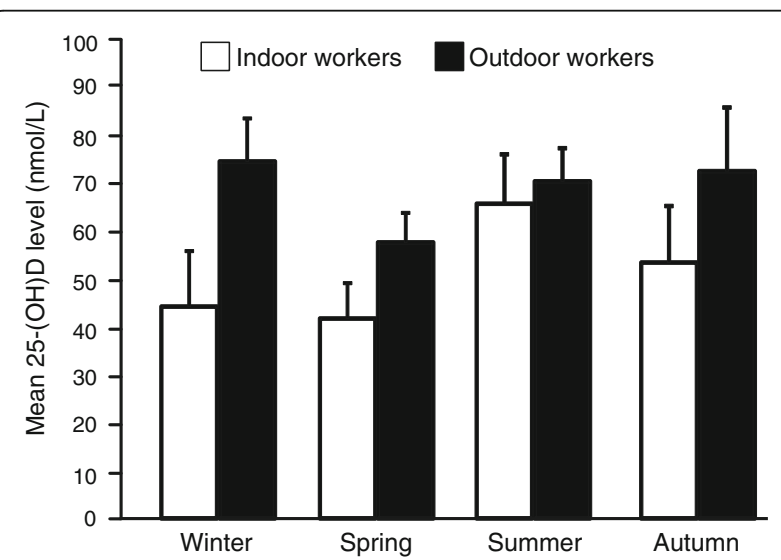

Fig. 3 Effect of seasons on 25-(OH)D level in indoor (white bars) and outdoor (black bars) workers. Data represent mean \pm standard error of the mean of each season for the given occupational group 
the level of summertime vitamin $\mathrm{D}$ among indoor workers were significantly different $(p<0.0001)$.

\section{General observations on the studies}

In general, serum 25-(OH)D levels in all occupational groups examined were below the optimal level as recommended by the ES, with the exception of lead/smelter workers. Lead/smelter workers had the highest level of serum vitamin $\mathrm{D}(77.8 \pm 5.4 \mathrm{nmol} / \mathrm{L})$ (Fig. 1) and were the only group whose average level was in the sufficient range. The average levels of vitamin $\mathrm{D}$ in outdoor workers, coalminers, practising physicians, nurses and other healthcare employees were in the insufficient range (25- $(\mathrm{OH}) \mathrm{D}$ of $50-75 \mathrm{nmol} / \mathrm{L})$ (Fig. 1). In indoor workers, shiftworkers, medical residents and healthcare students the average $25-(\mathrm{OH}) \mathrm{D}$ levels were in the deficient range $(\leq 50 \mathrm{nmol} / \mathrm{L})$. Average vitamin $\mathrm{D}$ levels were lowest $(33.8 \pm 10.1 \mathrm{nmol} / \mathrm{L})$ among shiftworkers (Fig. 1 and Table 1).

When the various occupational groups were analysed with regard to the percentage with vitamin D deficiency, $80 \%$ of shiftworkers were vitamin D deficient and this group demonstrated the largest proportion of subjects in the deficient range (Fig. 2). They were followed closely by indoor workers and healthcare students with $77 \%$ and $72 \%$ vitamin D deficiency, respectively. Overall, outdoor workers, practising physicians, nurses and other healthcare employees all had proportions with vitamin D deficiency below 50\% (Fig. 2).

The two occupational groups with the lowest proportion of combined deficiency or insufficiency were outdoor workers and other healthcare employees $(75 \%$ and $68 \%$, respectively) (Fig. 2). For practising physicians, about $96 \%$ were vitamin D deficient or insufficient. Likewise, indoor workers, shiftworkers and nurses all had about $90 \%$ deficiency or insufficiency.

The average serum vitamin $\mathrm{D}$ levels and prevalence of vitamin $D$ deficiency or insufficiency (Additional file 3: Figure S4A; Additional file 5: Figure S5A; Additional file 6: Figure S6A) were not dependent on geographical location.

\section{Discussion}

The global prevalence of vitamin D deficiency has reached an alarming proportion. This trend has elicited a significant amount of research interest to elucidate the potential causes of vitamin D deficiency and insufficiency in order to advance interventional strategies to ameliorate the associated risks $[2,46]$. Several studies have demonstrated that populations worldwide, including those located in sunny regions of the world, are at risk of vitamin D deficiency $[47,48]$. Some vulnerable demographic groups of the population, including pregnant women [49, 50], the elderly [51], hospitalized patients and other institutionalized groups $[12,52,53]$, and certain occupations $[45,54-56]$ who are predisposed to receive low or no exposure to sunshine, may be at a greater risk of developing vitamin D inadequacy.

In general, we found that vitamin D levels in most occupational groups fell well below the levels considered optimal for health [13, 57]. Additionally, we observed a higher prevalence of vitamin D deficiency in all occupational groups examined than the reported population burden of vitamin D deficiency in multiple populations, suggesting that workers may be particularly vulnerable to vitamin D deficiency [23, 41]. For instance, 25-(OH)D among US subpopulations demonstrated that adult women had mean serum 25-(OH)D levels of $62 \mathrm{nmol} / \mathrm{L}$ and $75 \mathrm{nmol} / \mathrm{L}$, respectively, in winter and summer [58]. Adult men had mean serum $25-(\mathrm{OH}) \mathrm{D}$ of $70 \mathrm{nmol} / \mathrm{L}$ and $82 \mathrm{nmol} / \mathrm{L}$ in winter and summer, respectively [58]. In contrast, Platz et al. [59] reported that among healthcare professionals in the US, the mean serum $25-(\mathrm{OH}) \mathrm{D}$ was $46 \mathrm{nmol} / \mathrm{L}$. The prevalence of vitamin D deficiency was 30\% [60] in the adult population in the US compared to $38 \%$ among medical residents [61, 62]. Other determinants of vitamin D deficiency include culture [63], geography $[64,65]$, genetics [66], disease states [67], diet [6] and age [68], and such other determinants will have to be considered alongside occupation as part of a comprehensive assessment of vitamin D status.

Among the occupations considered in the present study, indoor workers and shiftworkers demonstrated very low levels of serum $25-(\mathrm{OH}) \mathrm{D}$ and high rates of vitamin $\mathrm{D}$ deficiency and insufficiency. The relative risks of vitamin $\mathrm{D}$ deficiency and insufficiency in these two worker groups were also very high compared to the other worker groups. Indoor workers spend a high proportion of their working hours indoors without sunshine exposure. Additionally, indoor workers working conventional working hours would be expected to get their sunlight exposure during mornings and evenings, when sunlight intensity is relatively low. Since vitamin D is produced through sunshine and adequate UV exposure, sunlight deprivation in indoor workers may put them at greater risk of developing vitamin D deficiency and its accompanying health risks. Consistent with this assumption, our study showed that workers in an indoor setting displayed a lower level of vitamin D relative to their outdoor counterparts. Further, medical residents and healthcare students, who spend a considerable amount of time indoors, had vitamin D levels almost identical to the level of vitamin D observed in specified indoor workers (Fig. 1).

Our study also demonstrated that shiftworkers are at the highest risk of vitamin D deficiency or insufficiency when compared with other occupational groups. Shiftworkers make up about $20 \%$ of the workforce in developed 
countries [69]. Shift work may include rotational daytime shifts or overnight shifts. Kimlin and Tenkate [70] reported that workers with permanent night shifts receive less exposure to daytime solar UVB. This may result in a lower vitamin D level in shiftworkers relative to the general population. Additionally, shiftworkers with low sunlight exposure may depend on exogenous vitamin $\mathrm{D}$ for proportionately more of their total vitamin D requirements. These hypotheses are consistent with the findings of the present review, which revealed that shiftworkers had a low vitamin D level with a comparatively large proportion of workers with deficiency or insufficiency. The low level of serum 25-(OH)D seen in shiftworkers has been suggested to be associated with their predisposition to various diseases including cancers [71], musculoskeletal disorders, and cardiovascular disease [2, 72, 73].

The interpretation of our analysis is complicated by the fact that shiftworkers may work at various times of the day in a fixed or rotating pattern. Additionally, shiftworkers may have a greater proportion of their nonworking hours during daylight periods, and spend more time outdoors during nonworking days than indoor office workers. Another factor is that shiftworkers may be outdoor or indoor workers. Furthermore, shiftworkers may differ in dietary intake, use of vitamin D supplements, or other lifestyle factors from non-shiftworkers, and considerable variability may exist with regard to the amount of time spent outdoors. Nevertheless, a compelling finding from the present review is that shiftwork appears to be a strong predictor for vitamin $\mathrm{D}$ deficiency.

Another determining factor shown to impact vitamin D status in shiftworkers is the season during which vitamin D levels are measured. Ito et al. [28] demonstrated that, during the summer, the amount of ambient solar UVB can compensate for time confined working indoors in shiftworkers. However, in the winter, vitamin D levels were lower in shiftworkers who work fixed night shifts compared to the shiftworkers who work strictly daytime shifts. This finding was confirmed by Romano et al. [45], who showed that nighttime shiftworkers had lower vitamin D levels compared with daytime workers during spring. Taken together, these observations suggest that, although shiftworkers may be at greatest risk of vitamin D deficiency, spending sufficient time outside and obtaining sufficient UV exposure has the potential of alleviating this risk.

The lifestyle and nature of work of many healthcare professionals may suggest less opportunity to be exposed to daytime solar UVB. Thus, it is reasonable to assume that healthcare employees would be at risk of vitamin D deficiency. Additionally, working hours may be particularly long during the early training period for most healthcare professionals, where sun deprivation due to long working hours can be exacerbated by additional time spent indoors studying. Consistently, average vitamin D levels in healthcare students and medical residents were shown in the present review to be in the deficient range, and average serum vitamin $\mathrm{D}$ levels were significantly lower than those of practicing physicians, nurses, or other healthcare professionals. Indeed, the average serum vitamin D levels in medical residents and healthcare students were close to those of specified indoor workers. For healthcare professionals, our study revealed a high prevalence of vitamin D deficiency among healthcare students and medical residents. This is alarming, as students and residents are generally young adults, and vitamin $\mathrm{D}$ deficiency during early adulthood may decrease peak bone density and lead to an increased risk of osteopenia or osteoporosis in later life, as well as other long-term health impacts associated with suboptimal vitamin D status [74]. Additionally, vitamin D sufficiency in young healthcare professionals may be a surrogate marker for other healthy behaviours (e.g. outdoor exercise and good nutrition), and a high burden of vitamin $\mathrm{D}$ deficiency in trainees in the health disciplines should prompt enhanced educational measures on the importance of adequate vitamin $\mathrm{D}$, as well as an examination of the underlying training-related factors which may contribute to vitamin D deficiency.

Practising physicians, nurses and other healthcare workers had average serum vitamin D levels significantly higher than students and residents, although the average vitamin D levels in all three groups were still in the insufficient range. The prevalence of vitamin D deficiency or insufficiency was also very high among all healthcare professionals with the exception of the other healthcare employee group. The latter group comprised employees with diverse work environments and lifestyles, which suggests that some may have more exposure to solar UVB not considered typical of many healthcare professionals. This could account for the relatively low prevalence of vitamin D deficiency or insufficiency in the other healthcare employees group.

The large proportion of studies on healthcare workers may in part reflect the use of healthcare workers as a convenience sample of young, presumed healthy individuals. Additionally, several large population-based studies in health professionals (e.g. Nurses' Health Study, Physicians' Health Study) have been performed, and publications arising out of these cohorts are included in our analysis. However, it is concerning that among healthcare workers, such a high prevalence of vitamin D deficiency exists. This may reflect a number of occupational factors including long working hours mainly indoors $[61,62]$, shiftwork and a tendency for healthcare workers to neglect their own health $[29,75,76]$. The extent to 
which healthcare workers, in general, are aware of their own vitamin D status is unknown, and it is conceivable that targeted interventions aimed at identifying and treating vitamin $\mathrm{D}$ deficiency in health care workers may be beneficial. Of note, some healthcare professions (e.g. nursing) have a high proportion of female workers of childbearing age, and there may be ancillary health benefits beyond those to healthcare workers themselves.

Surprisingly, our data also demonstrate that among healthcare professionals, only healthcare students had an increased risk to develop vitamin D deficiency. However, all healthcare professionals had an elevated risk of vitamin $\mathrm{D}$ insufficiency, in keeping with the $25-(\mathrm{OH}) \mathrm{D}$ levels in these worker categories.

Lead exposure, either short- or long-term, has the potential to influence the metabolism of vitamin $\mathrm{D}$ [77, $78]$. The impact of lead on serum $1 \alpha, 25-(\mathrm{OH})_{2} \mathrm{D}$ levels is attributed to the inhibitory effect of lead on cytochrome $\mathrm{P} 450$ in the proximal tubules of the kidney, which mediates the hydroxylation of $25-(\mathrm{OH}) \mathrm{D}$ to the dihydroxy metabolite. In adults occupationally exposed to lead [78], Mason et al. demonstrated increased serum $1 \alpha, 25-(\mathrm{OH})_{2} \mathrm{D}$ levels in lead-exposed workers compared with a referent group who were not occupationally exposed to lead. In contrast, Greenberg et al. [79] did not demonstrate any effect of lead exposure on serum $1 \alpha$, $25-(\mathrm{OH})_{2} \mathrm{D}$ levels.

Of all the occupational groups considered in our study, lead/smelter workers had the highest level of circulating $25-(\mathrm{OH}) \mathrm{D}$. The mean $1 \alpha, 25-(\mathrm{OH})_{2} \mathrm{D}$ levels found in lead/ smelter workers in our study was $139.73 \pm 57.51 \mathrm{pmol} / \mathrm{L}$, consistent with the average serum $1 \alpha, 25-(\mathrm{OH})_{2} \mathrm{D}$ levels found in the general population [80]. This may imply that the lead/smelter workers did not demonstrate compromised renal hydroxylase activity. The number of studies on lead/smelter workers and the number of study participants were, however, very few relative to the other occupational groups examined except coalminers. Moreover, the studies were older and the nutrition of the subjects at the time may have been different compared to subjects in more recent studies. Furthermore, the studies on lead/smelter workers presented in this report also are of low quality; they did not demonstrate any relationship between serum 25- $(\mathrm{OH}) \mathrm{D}$ levels and blood lead levels. Thus, modern and high quality studies that account for all confounders of the relationship between lead exposure and serum 25-(OHD) and $1 \alpha, 25-$ $(\mathrm{OH})_{2} \mathrm{D}$ levels are warranted to establish if there is an association between lead exposure and vitamin $\mathrm{D}$ status.

Likewise, the literature is lacking in high quality studies that describe the relationship between coalminers and serum vitamin D levels. Our review found only three reports [81-83] comprising 314 subjects. Coalminers can be underground miners or surface miners. Underground miners experience reduced exposure to sunlight and, following on from the previous discussion, may be at increased risk of low vitamin D status compared to surface miners. Surprisingly, our findings revealed that vitamin $D$ status in underground miners was not significantly different from surface miners. The lack of appreciable difference in these two group of miners may mean that exposure of the underground group to sunlight in-between shifts could be sufficient to maintain serum vitamin D levels. In keeping with this view, Shuster et al. [81, 82] showed that, in the summer and winter seasons, serum vitamin D levels were not significantly different between underground and surface workers. However, in the summer months, serum vitamin D levels were higher than the corresponding levels seen during the winter months. These findings were in agreement with those demonstrated by Sarikaya et al. [83] in underground and surface miners.

The angle at which the sun rays impact the skin, which is a function of latitude, determines the amount of vitamin D production [64]. The more oblique the angle, the lesser the amount of vitamin D synthesized [84]. At latitudes beyond $35^{\circ}$, vitamin $\mathrm{D}$ production declines [65]. However, geography did not seem to be an obvious determinant of the difference in vitamin D level, as evidenced in the apparent lack of impact of latitude on vitamin D levels (Additional file 3: Figure S4A-C), and this is consistent with other published literature. A recent study in the US demonstrated that, for a large proportion of the year (March - October), serum vitamin D status was independent of geographical latitude [64]. This study further described that latitude becomes limiting only during the winter months (November - February). In our review, indoor vs. outdoor work and the amount of time exposed to sunlight seemed to be the dominant determinant of vitamin $\mathrm{D}$ levels.

Serum $25-(\mathrm{OH}) \mathrm{D}$ levels vary widely according to the season of the year in which the studies or collection of samples are conducted [25, 85], thus establishing season of the year as a confounder of serum vitamin D level [84]. Several lines of evidence suggest that there is a high variability in seasonal vitamin $\mathrm{D}$ levels across the globe [86, 87]. In a study on a normal Japanese population (adults without any abnormal biochemical data shown on routine medical check-up; in particular, those not suffering from parathyroid or calcium-related diseases, based on biochemical measurements and clinical assessment), Ono et al. [88] showed that mean serum 25$(\mathrm{OH}) \mathrm{D}$ levels were lowest in winter and spring, and peaked in the summer and the beginning of autumn. These findings were consistent with data from healthy postmenopausal women in New Zealand presented by Bolland et al. [89]. Accordingly, vitamin D deficiency was more prevalent in the spring and winter relative to the level of deficiency seen in summer and autumn [64]. 
These findings confirm the dependence of vitamin D status on season. Devgun et al. [85] also demonstrated in both indoor and outdoor workers that serum 25$(\mathrm{OH}) \mathrm{D}$ levels varied significantly according to season, being lowest in the spring and winter, and highest in the late summer and the beginning of autumn, in agreement with previous data [88, 89]. More importantly, they showed that vitamin $\mathrm{D}$ levels in outdoor workers were higher relative to indoor workers for all seasons but more pronounced in early winter.

In this systematic review, we could not perform a meta-analysis on all occupational groups to determine the effect of seasonality on vitamin D status because most of the included studies did not report on the season in which serum vitamin $\mathrm{D}$ level was assessed. We suggest that seasonality should be routinely reported in future studies on vitamin D levels to try to address this gap. Nonetheless, from the included studies which reported on seasons in indoor and outdoor workers, our data showed that vitamin D levels in outdoor workers were higher relative to indoor workers in all four seasons (Fig. 3). An unexpected finding in the present study was that the level of 25$(\mathrm{OH}) \mathrm{D}$ in the winter was comparable to the summer and autumn values in outdoor workers. Endogenous vitamin $\mathrm{D}$ synthesis is a function of UV radiation in the wavelength range of $280-320 \mathrm{~nm}$, which in turn depends on season and latitude $[64,65,85,90]$. At high latitudes $\left(>35^{\circ} \mathrm{N}\right)$ UV radiation becomes almost negligible in the winter months, which consequently affects vitamin D production [85]. In contrast, at lower latitudes, UV radiation is not limiting during the winter months, which suggests that vitamin D synthesis can proceed all year long [64]. An alternative explanation may be that it is possible to accumulate sufficient vitamin $\mathrm{D}$ stores to get through the winter, but that the stores are depleted by springtime. Therefore, the lowest levels are seen in spring.

In the present study, the location of the studies that contributed the highest amount to the pooled mean 25$(\mathrm{OH}) \mathrm{D}$ level in outdoor workers in the winter were at latitudes $<35^{\circ} \mathrm{N}$ [91]. Thus, outdoor workers in these regions may not experience huge seasonal variation in vitamin D synthesis compared to indoor workers. Together with the fact that there were few studies that contributed to the analysis of serum 25- $(\mathrm{OH}) \mathrm{D}$ level dependence on season, this could account for the relatively high level of 25-(OH)D in outdoor workers during the winter season.

Most adults in the general population globally have vitamin D inadequacy $[13,46]$. A significant proportion of the adult population in Europe, the US and Canada have vitamin $\mathrm{D}$ deficiency $[2,92]$. Despite the relationship between sunlight exposure and vitamin D levels, vitamin D deficiency is reportedly also prevalent among populations living in sunny climates including the Middle East, Africa, Australia, India and South America [2, 93, 94]. Population-level prevalences of vitamin D deficiency have been reported as 59\% in the Canadian population [95], 52\% in the Danish population [96], and 40\% in the US population [97, 98]. Our systematic review suggests that occupation is a major determinant that may contribute to suboptimal vitamin D levels and that workers in some occupations have lower average levels of vitamin D and a higher prevalence of deficiency compared to the general public. Indoor workers, shiftworkers, medical residents, healthcare students, practising physicians and coalminers have a particularly high prevalence of vitamin D deficiency. However, most occupational groups considered in this review, with the exception of lead/smelter workers, had a moderate to high burden of vitamin D deficiency or insufficiency.

\section{Clinical Implications and Recommendations}

Although population-wide vitamin D deficiency is a global phenomenon, from the present systematic review it is clear that workers in some occupational categories are at a greater risk for vitamin D deficiency than others. Regular screening for vitamin D levels in shiftworkers and other specific groups of workers should be considered for future clinical practice guidelines and population health initiatives, while existing workplace wellness programs should incorporate education about the importance of adequate vitamin $\mathrm{D}$ levels, sunlight exposure and adequate nutritional intake of vitamin D-rich foods to prevent adverse health outcomes related to vitamin D deficiency. Additionally, for occupations predominantly based indoors, workers could, where appropriate, be encouraged to take intermittent breaks outside to expose the skin to UV light in order to promote cutaneous vitamin D synthesis $[99,100]$, and work schedules could be re-imagined to allow for such breaks, while of course avoiding excessive sunlight exposure.

\section{Limitations}

To further the aim of a robust body of literature on the health effects of suboptimal vitamin D status, the academic community would benefit from a consensus as to what constitutes vitamin D deficiency. Due to an existing lack of agreement on the definition of vitamin D deficiency, combining data from studies where the study authors have used different definitions of adequate vitamin D status is challenging. In the present study, we used the ES's definition of vitamin D deficiency and insufficiency. Other approaches, such as using the Institute of Medicine's definitions, could also be justified. Agreement on what constitutes vitamin D insufficiency and deficiency will additionally enhance standardization of 
guidelines and interventional efforts targeted at at-risk occupational groups in the population.

Another limitation is the methodology employed in assaying serum vitamin D levels. In the present review, the majority of the included studies used the RIA technique to evaluate vitamin D status. Several lines of evidence have shown that there is a marked inter-laboratory variation in results obtained with this assay type, which could be as high as $30 \%[8,13]$. These variations need to be borne in mind when pooling data from different studies.

Serum vitamin D level is determined by latitude (geographical location), season (UVB), cultural traditions (clothing), diet and sex. The present review combines studies conducted at different latitudes, seasons and with subjects from diverse cultural backgrounds. This has the potential to overestimate or underestimate the influence of occupation on vitamin $\mathrm{D}$. These differences may also create a high degree of heterogeneity between individual studies making conclusions derived from pooled data less reliable.

Our systematic review is also limited with regard to occupational detail, as we relied on what was reported by the primary study authors in their description of the subjects' occupations or occupational categories.

\section{Recommendations for future studies}

Based on the above limitations, we suggest that future studies measuring vitamin D status employ assay techniques with minimal inter-laboratory variations. One method, which has been shown to be consistently reproducible, is liquid chromatography [101]. In a review to compare different assays used to assess vitamin D status, it was demonstrated that liquid chromatography followed by tandem mass spectrometry produced the lowest variability across different laboratories [6]. It is recommended that the assay technique for the assessment of vitamin D levels should be standardized to enable ready comparison and meta-analysis.

Though medical students and residents may still be considered as a convenience sample, future studies employing this group as subjects should not presume that they are necessarily a population of "healthy" young adults.

Since season of the year is a major determinant of vitamin D levels, future studies should comment on the season in which the study is performed.

Future studies should also incorporate additional confounders such as measures of sunlight exposure and diet. Studies on shiftworkers should furthermore provide an indication of the type of shiftwork performed.

\section{Conclusions}

Individuals who work predominantly indoors and shiftworkers are at risk of developing vitamin deficiency or insufficiency. Despite a lack of consensus on optimal levels of vitamin $\mathrm{D}$ for health, vitamin D insufficiency and deficiency are common in the occupational groups investigated, and some workers should be considered an at-risk group for vitamin D deficiency. Further high quality studies are needed to explore the relationship between occupation and vitamin D status. The assumption that trainees in the health care disciplines represent a convenience sample of "healthy" adults may not always be true. Guidelines on screening for vitamin D deficiency and supplementation strategies in vulnerable groups should include consideration of occupation.

\section{Additional files}

\begin{abstract}
Additional file 1: Search strategies. (DOCX $21 \mathrm{~kb}$ )
Additional file 2: Study selection. (DOC $57 \mathrm{~kb}$ )

Additional file 3: 25-(OH)D levels. Figure S4A. Effect of latitude on serum $25-(\mathrm{OH}) \mathrm{D}$ levels in all occupational groups examined. Each data point represents mean \pm standard error of the mean of each included study. Inset: symbols and colors of each occupational subgroup. N/S: Northern/Southern hemisphere. Figure S4B. Comparison of serum 25$(\mathrm{OH}) \mathrm{D}$ levels in indoor and outdoor workers according to latitude. Each data point represents mean \pm standard error of the mean of studies of outdoor and indoor workers included in the analysis. Inset: symbols and colors of each occupational subgroup. N/S: Northern/Southern hemisphere. Figure S4C. Comparison of serum 25-(OH)D levels in healthcare workers according to latitude. Each data point represents mean \pm standard error of the mean of studies of each healthcare workers included in the analysis. Inset: symbols and colors of each occupational subgroup. N/S: Northern/Southern hemisphere. (ZIP 229 kb)
\end{abstract}

Additional file 4: 25-(OH)D levels in indoor and outdoor workers. Figure S1. 25-(OH)D levels in indoor and outdoor workers. Data represent pooled weighted mean \pm pooled standard error of the mean for each group. ${ }^{*}$ Statistically significant compared with indoor workers $(p<0.0001)$. (PDF $212 \mathrm{~kb})$

Additional file 5: Latitude and \% vitamin D deficiency. Figure S5A. Effect of latitude on \% vitamin D deficiency in all the occupational groups included in analysis. Percent vitamin D deficiency was defined as the number of subjects of a particular study with mean 25-(OH)D levels less than $50 \mathrm{nmol} / \mathrm{L}$. Inset: symbols and colors of each occupational subgroup. N/S: Northern/Southern hemisphere.

Figure S5B. Effect of latitude on \% vitamin D deficiency in indoor and outdoor workers included in the analysis. Inset: symbols and colors of each occupational subgroup. N/S: Northern/Southern hemisphere. Figure S5C. Effect of latitude on \% vitamin D deficiency in healthcare employees included in the analysis. Inset: symbols and colors of each occupational subgroup. N/S: Northern/Southern hemisphere. (ZIP 195 kb)

Additional file 6: Latitude and \% vitamin D deficiency or insufficiency. Figure S6A. Effect of latitude on \% vitamin D deficiency or insufficiency in all occupational groups examined. Percent vitamin D insufficiency was defined as the number of subjects of a particular study with mean 25$(\mathrm{OH}) \mathrm{D}$ levels less than $75 \mathrm{nmol} / \mathrm{L}$. Inset: symbols and colors of each occupational group. N/S: Northern/Southern hemisphere. Figure S6B. Effect of latitude on \% vitamin D deficiency or insufficiency in indoor and outdoor workers. Inset: symbols and colors of each occupational subgroup. N/S: Northern/Southern hemisphere. Figure S6C. Effect of latitude on \% vitamin D deficiency or insufficiency in healthcare professionals. Inset: symbols and colors of each occupational subgroup. N/S: Northern/Southern hemisphere. (ZIP $189 \mathrm{~kb}$ )

Additional file 7: $25-(\mathrm{OH}) \mathrm{D}$ levels in underground and surface coalminers. Figure S2. 25-(OH)D levels in underground and surface coalminers. Data represent pooled weighted mean \pm pooled standard error of the mean for each group. NS, no significant difference. (PDF $213 \mathrm{~kb})$ 
Additional file 8: $25-(\mathrm{OH}) \mathrm{D}$ levels in healthcare professionals. Figure S3. 25- $(\mathrm{OH}) \mathrm{D}$ levels among different healthcare professionals. Data represent pooled weighted mean \pm pooled standard error of the mean for each healthcare category. * Statistically significant compared to medical residents $(p<0.05)$

(PDF $222 \mathrm{~kb}$ )

\section{Abbreviations}

1a, 25-(OH) 2 D: 1a, 25-dihydroxyvitamin D; 25- $(\mathrm{OH}) \mathrm{D}$ : 25-hydroxyvitamin $\mathrm{D}$; Cl: Confidence interval; CV: Coefficient of variation; ElA: Enzyme immunoassay; ELISA: Enzyme-linked immunosorbent assay; ES: Endocrine Society; Fig.: Figure; HPLC: High performance liquid chromatography; IQR: Interquartile range; I: Liter; ml : Milliliter; N: North; ng: Nanogram; nmol: Nanomole; pmol: Picomole; RIA: Radioimmunoassay; RR: Relative risk; SD: Standard deviation; SE: Standard error of the mean; UVB: Ultraviolet B

\section{Acknowledgements}

The authors wish to acknowledge Robert Boyko for help with the figures.

\section{Funding}

This work was supported by institutional funding from the Department of Medicine, University of Alberta to Sebastian Straube.

\section{Availability of data and materials}

All data that we used for this systematic review are in the public domain.

\section{Authors' contributions}

Sebastian Straube and Xiangning Fan conceived of the idea of this systematic review. Liz Dennett did the literature searching. Daniel Sowah and Xiangning Fan decided on study eligibility and performed the data extraction, under the guidance of Sebastian Straube, who resolved disagreements where needed. Reidar Hagtvedt provided statistical expertise. Daniel Sowah and Xiangning Fan performed the analyses, drafted the manuscript, and made the figures. All authors contributed to the revision of the manuscript and approved the final version for submission.

\section{Competing interests}

Daniel Sowah has no interests to declare. Xiangning Fan has no interests to declare. Liz Dennett has no interests to declare. Reidar Hagtvedt has no interests to declare. Sebastian Straube declares honoraria from Oxford Medical Knowledge and advisory board fees from Daiichi Sankyo, Inc.; all unrelated to the topic of this review.

\section{Consent for publication}

Not applicable as this is a systematic review.

\section{Ethics approval and consent to participate}

Not applicable as this is a systematic review.

\section{Publisher's Note}

Springer Nature remains neutral with regard to jurisdictional claims in published maps and institutional affiliations.

\section{Author details}

'Division of Preventive Medicine, Department of Medicine, University of Alberta, 5-30 University Terrace, 8303-112 Street, Edmonton, AB T6G 2T4, Canada. ${ }^{2}$ JW Scott Health Sciences Library, University of Alberta, Edmonton, Canada. ${ }^{3}$ Department of Accounting, Operations and Information Systems, School of Business, University of Alberta, Edmonton, Canada.

Received: 22 August 2016 Accepted: 15 May 2017

Published online: 22 June 2017

\section{References}

1. Christakos S, Dhawan P, Liu Y, Peng X, Porta A. New insights into the mechanisms of vitamin D action. J Cell Biochem. 2003:88(4):695-705.

2. Holick MF. Vitamin D deficiency. N Engl J Med. 2007;357(3):266-81.
3. Grober U, Spitz J, Reichrath J, Kisters K, Holick MF. Vitamin D: Update 2013 From rickets prophylaxis to general preventive healthcare.

Dermatoendocrinol. 2013:5(3):331-47.

4. Straube S, Andrew Moore R, Derry S, McQuay HJ. Vitamin D and chronic pain Pain. 2009;141(1-2):10-3.

5. Adams JS, Hewison M. Update in vitamin D. J Clin Endocrinol Metab. 2010; 95(2):471-8.

6. Fuleihan G, Bouillon R, Clarke B, Chakhtoura M, Cooper C, McClung $M$, et al. Serum 25-hydroxyvitamin D levels: variability, knowledge gaps, and the concept of a desirable range. J Bone Miner Res. 2015; 30(7):1119-33

7. Awada Z, Ossaily S, Zgheib N. The nutrigenetics and pharmacogenetics of vitamin D pathways. Curr Pharmacogenomics Person Med. 2014;12(2):89-103.

8. Holick MF, Binkley NC, Bischoff-Ferrari HA, Gordon CM, Hanley DA, Heaney RP, et al., Endocrine Society. Evaluation, treatment, and prevention of vitamin D deficiency: an Endocrine Society clinical practice guideline. J Clin Endocrinol Metab. 2011:96(7):1911-30.

9. Lehmann B, Meurer M. Extrarenal sites of calcitriol synthesis: the particular role of the skin. Recent Results Cancer Res. 2003;164:135-45.

10. Institute of Medicine (IOM) Committee, Ross AC, Taylor CL, Yaktine AL, Del Valle HB. Dietary Reference Intakes for Vitamin D and Calcium. Washington, DC: The National Academies Press; 2011.

11. LeBlanc ES, Zakher B, Daeges M, Pappas M, Chou R. Screening for vitamin D deficiency: a systematic review for the U.S. Preventive Services Task Force. Ann Intern Med. 2015;162(2):109-22.

12. Hilger J, Friedel A, Herr R, Rausch T, Roos F, Wahl DA, et al. A systematic review of vitamin D status in populations worldwide. Br J Nutr. 2014;111(1):23-45

13. van Schoor NM, Lips P. Worldwide vitamin D status. Best Pract Res Clin Endocrinol Metab. 2011:25(4):671-80.

14. Williams C. Work-life balance of shift workers. Perspectives. Statistics Canada August 2008, Statistics Canada Catalogue no. 75-001-X:5-6-16.

15. TM MM. A time to work: recent trends in shift work and flexible schedules, Monthly Labor Review. Washington, DC: Bureau of Labor Statistics; 2007

16. International Labour Office, Conditions of Work and Employment Programme. Shift work. Geneva: International Labour Organization; 2004. p. Information Sheet No. WT-8.

17. Harrington J. Health effects of shift work and extended hours of work. Occup Environ Med. 2001:58(1):68-72.

18. Knutsson A. Health disorders of shift workers. Occup Med. 2003;53(2):103-8.

19. Costa-Fernandes N, Adodra A, Blair M, Kwong H. Awareness, knowledge and practice of vitamin d deficiency amongst health care professionals in northwest London. Arch Dis Child. 2014;99:A25.

20. Fritschi L, Erren TC, Glass DC, et al. The association between different night shiftwork factors and breast cancer: a case-control study. Br J Cancer. 2013; 109(9):2472-80.

21. Mendell MJ, Fisk WJ, Kreiss K, Levin H, Alexander D, Cain WS, et al. Improving the health of workers in indoor environments: Priority research needs for a National Occupational Research Agenda. Am J Public Health. 2002:92(9):1430-40.

22. Olivieri M, Biscardo CA, Valenza D, Verlato G. Night shift, vitamin D and occupational allergies in bakers. Eur Respir J. 2014:44(Suppl 58):P4539.

23. Jeong H, Hong S, Heo Y, Chun H, Kim D, Park J, et al. Vitamin D status and associated occupational factors in Korean wage workers: data from the 5th Korea national health and nutrition examination survey (KNHANES 20102012). Ann occup environ med. 2014;26:28.

24. Sari DK, Damanik HA, Lipoeto NI, Lubis Z. Low serum 25(OH)D levels are associated with single nucleotide polymorphisms of the vitamin $D$ receptor gene and lifestyle factors, especially in women with higher body fat percentage. Obes Res and Clin Pract. 2013;7:12-3.

25. Devgun MS, Paterson CR, Martin BT. Seasonal changes in the activity of serum alkaline phosphatase. Enzyme. 1981;26(6):301-5

26. Nakamura K, Nashimoto M, Hori Y, Muto K, Yamamoto M. Serum 25hydroxyvitamin $\mathrm{D}$ levels in active women of middle and advanced age in a rural community in Japan. Nutrition. 1999:15(11-12):870-3.

27. Bachhel $R$, Singh NR, Sidhu JS. Prevalence of vitamin D deficiency in north-west Punjab population: A cross-sectional study. Int J Appl Basic Med Res. 2015;5(1):7-11.

28. Itoh H, Weng Z, Saito H, Ogawa Y, Nakayama K, Hasegawa-Ohira M, et al. Association between night-shift work and serum 25-hydroxyvitamin D levels 
in Japanese male indoor workers: a cross-sectional study. Ind Health. 2011; 49(5):658-62.

29. Beloyartseva M, Mithal A, Kaur P, Kalra S, Baruah MP, Mukhopadhyay S, et al. Widespread vitamin D deficiency among Indian health care professionals. Arch Osteoporos. 2012;7(1-2):187-92.

30. Latitude/longitude finder - MY NASA DATA. [https://mynasadata.larc.nasa. gov/latitudelongitude-finder/]. Accessed 6 June 2016.

31. Binkley N, Krueger D, Cowgill CS, Plum L, Lake E, Hansen KE, et al. Assay variation confounds the diagnosis of hypovitaminosis D: a call for standardization. J Clin Endocrinol Metab. 2004;89(7):3152-7.

32. Barake M, Daher RT, Salti I, Cortas NK, Al-Shaar L, Habib RH, et al. 25hydroxyvitamin D assay variations and impact on clinical decision making. J Clin Endocrinol Metab. 2012;97(3):835-43.

33. Carter GD. Accuracy of 25-hydroxyvitamin D assays: confronting the issues. Curr Drug Targets. 2011;12(1):19-28.

34. Table 7.7.a. Formulae for combining groups. [http://handbook.cochrane.org/ chapter_7/table_7_7_a_formulae_for_combining_groups.htm]. Accessed 6 June 2016.

35. Pooled Standard Error versus the Satterthwaite Approximation. [http:// wolfweb.unr.edu/ ldyer/classes/396/PSE.pdf]. Accessed 6 June 2016.

36. Wan $X$, Wang W, Liu J, Tong T. Estimating the sample mean and standard deviation from the sample size, median, range and/or interquartile range. BMC Med Res Methodol. 2014;14:135.

37. Hozo S, Djulbegovic B, Hozo I. Estimating the mean and variance from the median, range, and the size of a sample. BMC Med Res Methodol. 2005;5(1):13.

38. Bland M. Estimating mean and standard deviation from the sample size, three quartiles, minimum, and maximum. Int J Stat Med Res. 2015:4:57-64.

39. Chalkley SR, Richmond J, Barltrop D. Measurement of vitamin D3 metabolites in smelter workers exposed to lead and cadmium. Occup Environ Med. 1998;55(7):446-52.

40. Mizoue T, Kochi T, Akter S, Eguchi M, Kurotani K, Tsuruoka H, et al. Low serum 25-hydroxyvitamin D concentrations are associated with increased likelihood of having depressive symptoms among Japanese workers. J Nutr. 2015;145(3):541-6

41. Ward M, Berry DJ, Power C, Hypponen E. Working patterns and vitamin D status in mid-life: a cross-sectional study of the 1958 British birth cohort. Occup Environ Med. 2011;68(12):902-7.

42. Pham NM, Akter S, Kurotani K, Nanri A, Sato M, Hayabuchi H, et al. Serum25hydroxyvitamin $\mathrm{D}$ and markers of insulin resistance in a Japanese working population. Eur J Clin Nutr. 2012;66(12):1323-8.

43. 7.41.3.8: Combining groups. [http://handbook.cochrane.org/chapter_7/7_7_ 3_8_combining_groups.htm]. Accessed 6 June 2016.

44. Heaney RP. Functional indices of vitamin D status and ramifications of vitamin D deficiency. Am J Clin Nutr. 2004;80(6 Suppl):1706S-9S.

45. Romano A, Vigna L, Belluigi V, Conti DM, Barberi CE, Tomaino L, et al. Shift work and serum $25-\mathrm{OH}$ vitamin D status among factory workers in Northern Italy: Cross-sectional study. Chronobiol Int. 2015; 32(6):842-7.

46. Lips P. Worldwide status of vitamin D nutrition. J Steroid Biochem Mol Biol. 2010;121(1-2):297-300

47. Gonzalez G, Alvarado JN, Rojas A, Navarrete C, Velasquez CG, Arteaga E. High prevalence of vitamin $D$ deficiency in Chilean healthy postmenopausal women with normal sun exposure: additional evidence for a worldwide concern. Menopause. 2007;14(3 Pt 1):455-61.

48. van der Meer IM, Middelkoop BJ, Boeke AJ, Lips P. Prevalence of vitamin D deficiency among Turkish, Moroccan, Indian and sub-Sahara African populations in Europe and their countries of origin: an overview. Osteoporos Int. 2011;22(4):1009-21.

49. Sachan A, Gupta R, Das V, Agarwal A, Awasthi PK, Bhatia V. High prevalence of vitamin $D$ deficiency among pregnant women and their newborns in northern India. Am J Clin Nutr. 2005;81(5):1060-4.

50. Ginde AA, Sullivan AF, Mansbach JM, Camargo CA Jr. Vitamin D insufficiency in pregnant and nonpregnant women of childbearing age in the United States. Am J Obstet Gynecol. 2010;202(5):436. e1-436.e8.

51. Toss $G$, Almqvist $S$, Larsson L, Zetterqvist H. Vitamin D Deficiency in Welfare Institutions for the Aged. Acta Med Scand. 2009;208(1-6):87-9.

52. McKenna MJ, Freaney R, Meade A, Muldowney FP. Hypovitaminosis D and elevated serum alkaline phosphatase in elderly Irish people. Am J Clin Nutr. 1985;41(1):101-9

53. Portela ML, Monico A, Barahona A, Dupraz H, Sol Gonzales-Chaves MM, Zeni SN. Comparative 25-OH-vitamin D level in institutionalized women older than 65 years from two cities in Spain and Argentina having a similar solar radiation index. Nutrition. 2010;26(3):283-9.

54. Kwon SI, Son JS, Kim YO, Chae CH, Kim JH, Kim CW, et al. Association between serum vitamin $\mathrm{D}$ and depressive symptoms among female workers in the manufacturing industry. Ann Occup Environ Med. 2015;27:28.

55. Roomi MA, Farooq A, Ullah E, Lone KP. Hypovitaminosis D and its association with lifestyle factors. Pak J Med Sci. 2015;31(5):1236-40.

56. Xiang F, Jiang J, Li H, Yuan J, Yang R, Wang Q, et al. High prevalence of vitamin $D$ insufficiency in pregnant women working indoors and residing in Guiyang, China. J Endocrinol Invest. 2013;36(7):503-7.

57. Ross AC, Manson JE, Abrams SA, Aloia JF, Brannon PM, Clinton SK, et al. The 2011 Dietary Reference Intakes for Calcium and Vitamin D: What Dietetics Practitioners Need to Know. J Am Diet Assoc. 2011;111(4):524-7.

58. Looker AC, Dawson-Hughes B, Calvo MS, Gunter EW, Sahyoun NR. Serum 25-hydroxyvitamin D status of adolescents and adults in two seasonal subpopulations from NHANES III. Bone. 2002;30(5):771-7.

59. Platz EA, Rimm EB, Willett WC, Kantoff PW, Giovannucci E. Racial variation in prostate cancer incidence and in hormonal system markers among male health professionals. J Natl Cancer Inst. 2000;92(24):2009-17.

60. Schleicher RL, Sternberg MR, Lacher DA, Sempos CT, Looker AC, Durazo-Arvizu RA, et al. The vitamin D status of the US population from 1988 to 2010 using standardized serum concentrations of 25hydroxyvitamin D shows recent modest increases. Am J of Clin Nutr. 2016;104(2):454-61.

61. Haney EM, Stadler D, Bliziotes MM. Vitamin D Insufficiency in Internal Medicine Residents. Calcif Tissue Int 2004. 2005;76(1):11-6.

62. Martineau AR, Hanifa Y, Witt KD, Barnes NC, Hooper RL, Patel M, et al. Double-blind randomised controlled trial of vitamin D3 supplementation for the prevention of acute respiratory infection in older adults and their carers (ViDiFlu). Thorax. 2015:70(10):953-60.

63. Batieha A, Khader $Y$, Jaddou H, Hyassat D, Batieha Z, Khateeb M, et al. Vitamin D status in Jordan: dress style and gender discrepancies. Ann Nutr Metab. 2011;58(1):10-8.

64. Kimlin MG, Olds WJ, Moore MR. Location and Vitamin D synthesis: Is the hypothesis validated by geophysical data? J Photochem Photobiol B Biol. 2007:86(3):234-9.

65. Kimlin MG. Geographic location and vitamin D synthesis. Mol Asp Med. 2008;29(6):453-61.

66. Wang TJ, Zhang F, Richards JB, Kestenbaum B, van Meurs JB, Berry D, et al. Common genetic determinants of vitamin $D$ insufficiency: a genome-wide association study. Lancet. 2010;376(9736):180-8.

67. Zhou C, Assem M, Tay JC, Watkins PB, Blumberg B, Schuetz EG, et al. Steroid and xenobiotic receptor and vitamin D receptor crosstalk mediates CYP24 expression and drug-induced osteomalacia. J Clin Invest. 2006;1 16(6):1703-12.

68. MacLaughlin J, Holick MF. Aging decreases the capacity of human skin to produce vitamin D3. J Clin Invest. 1985;76(4):1536-8.

69. Erren TC, Morfeld P, Stork J, Knauth P, von Mulmann MJ, Breitstadt R, et al. Shift work, chronodisruption and cancer?-The IARC 2007 challenge for research and prevention and 10 theses from the Cologne Colloquium 2008. Scand J Work Environ Health. 2009;35(1):74-9.

70. Kimlin MG, Tenkate TD. Occupational exposure to ultraviolet radiation: the duality dilemma. Rev Environ Health. 2007;22(1):1-37.

71. Fritschi L, Glass DC, Heyworth JS, Aronson K, Girschik J, Boyle T, et al. Hypotheses for mechanisms linking shiftwork and cancer. Med Hypotheses. 2011;77(3):430-6.

72. Freedman DM, Cahoon EK, Rajaraman P, Major JM, Doody MM, Alexander $\mathrm{BH}$, et al. Sunlight and other determinants of circulating 25-hydroxyvitamin D levels in black and white participants in a nationwide US study. Am J Epidemiol. 2013;177(2):180-92.

73. Lee JH, Gadi R, Spertus JA, Tang F, O'Keefe JH. Prevalence of vitamin D deficiency in patients with acute myocardial infarction. Am J Cardiol. 2011; 107(11):1636-8.

74. Holick MF. Sunlight and vitamin D for bone health and prevention of autoimmune diseases, cancers, and cardiovascular disease. Am J Clin Nutr. 2004;80(6 Suppl):1678S-88S.

75. Mahdy S, Al-Emadi SA, Khanjar IA, Hammoudeh MM, Sarakbi HA, Siam AM, et al. Vitamin D status in health care professionals in Qatar. Saudi Med J. 2010;31(1):74-7. 
76. Multani SK, Sarathi V, Shivane V, Bandgar TR, Menon PS, Shah NS. Study of bone mineral density in resident doctors working at a teaching hospital. J Postgrad Med. 2010;56(2):65-70.

77. Kristal-Boneh E, Froom P, Yerushalmi N, Harari G, Ribak J. Calcitropic hormones and occupational lead exposure. Am J Epidemiol. 1 998;147(5):458-63.

78. Mason HJ, Somervaille LJ, Wright AL, Chettle DR, Scott MC. Effect of occupational lead exposure on serum 1,25-dihydroxyvitamin D levels. Hum Exp Toxicol. 1990;9(1):29-34.

79. Greenberg A, Parkinson DK, Fetterolf DE, Puschett JB, Ellis KJ, Wielopolski L, et al. Effects of elevated lead and cadmium burdens on renal function and calcium metabolism. Arch Environ Health. 1986;41(2):69-76.

80. Stern PH, De Olazabal J, Bell NH. Evidence for abnormal regulation of circulating 1 alpha, 25-dihydroxyvitamin D in patients with sarcoidosis and normal calcium metabolism. J Clin Invest. 1980;66(4):852-5.

81. Shuster S, Chadwick L, Afacan AS, Robertson MD. Serum 25-hydroxy vitamin $D$ in coalworkers and surface workers in winter. Br Med J. 1982;284(6313):386.

82. Shuster $S$, Chadwick L, Afacan AS, Robertson MD. Serum 25-hydroxy vitamin $\mathrm{D}$ in surface and underground coalminers. Br Med J (Clin Res Ed). 1981;283(6284):106.

83. Sarikaya S, Ozdolap S, Mungan AG, Gumustas S, Koc U, Guven B, et al. Effect of underground working on vitamin $D$ levels and bone mineral densities in coal miners: a controlled study. J Int Med Res. 2006;34(4):362-6.

84. Webb AR, Kline L, Holick MF. Influence of season and latitude on the cutaneous synthesis of vitamin D3: exposure to winter sunlight in Boston and Edmonton will not promote vitamin D3 synthesis in human skin. J Clin Endocrinol Metab. 1988;67(2):373-8.

85. Devgun MS, Paterson CR, Johnson BE, Cohen C. Vitamin D nutrition in relation to season and occupation. Am J Clin Nutr. 1981:34(8):1501-4.

86. Marwaha RK, Yenamandra VK, Sreenivas V, Sahay R, Baruah MP, Desai A, et al. Regional and seasonal variations in ultraviolet B irradiation and vitamin D synthesis in India. Osteoporos Int. 2016;27(4):1611-7.

87. Engelsen $\mathrm{O}$, Brustad M, Aksnes L, Lund E. Daily duration of vitamin D synthesis in human skin with relation to latitude, total ozone, altitude, ground cover, aerosols and cloud thickness. Photochem Photobiol. 2005; 81(6):1287-90

88. Ono Y, Suzuki A, Kotake M, Zhang X, Nishiwaki-Yasuda K, Ishiwata Y, et al. Seasonal changes of serum 25 -hydroxyvitamin $D$ and intact parathyroid hormone levels in a normal Japanese population. J Bone Miner Metab. 2005;23(2):147-51.

89. Bolland MJ, Grey AB, Ames RW, Mason BH, Horne AM, Gamble GD, et al. The effects of seasonal variation of 25-hydroxyvitamin $D$ and fat mass on a diagnosis of vitamin D sufficiency. Am J Clin Nutr. 2007;86(4):959-64.

90. Stamp TC, Round JM. Seasonal changes in human plasma levels of 25hydroxyvitamin D. Nature. 1974;247(5442):563-5.

91. Azizi E, Pavlotsky F, Kudish A, Flint P, Solomon A, Lerman Y, et al. Serum levels of 25-hydroxy-vitamin D3 among sun-protected outdoor workers in Israel. Photochem Photobiol. 2012;88(6):1507-12.

92. Harkness LS, Cromer BA. Vitamin D deficiency in adolescent females. J Adolesc Health. 2005;37(1):75

93. Marwaha RK, Tandon N, Shivaprasad C, Kanwar R, Mani K, Aggarwal R, et al. Peak bone mineral density of physically active healthy Indian men with adequate nutrition and no known current constraints to bone mineralization. J Clin Densitom. 2009;12(3):314-21.

94. Thacher TD, Clarke BL. Vitamin D insufficiency. Mayo Clin Proc. 2011;86(1):50-60.

95. Greene-Finestone LS, Berger C, de Groh M, Hanley DA, Hidiroglou N, Sarafin K, et al., CaMos Research Group. 25-Hydroxyvitamin D in Canadian adults: biological, environmental, and behavioral correlates. Osteoporos Int. 2011;22(5):1389-99.

96. Thuesen B, Husemoen L, Fenger M, Jakobsen J, Schwarz P, Toft U, et al. Determinants of vitamin $D$ status in a general population of Danish adults. Bone. 2012:50(3):605-10.

97. Forrest KY, Stuhldreher WL. Prevalence and correlates of vitamin D deficiency in US adults. Nutr Res. 2011;31(1):48-54.

98. Janz T, Pearson C. Vitamin D blood levels of Canadians. Statistics Canada Catalogue no. 82-624-X. [http://www.statcan.gc.ca/pub/82-624-x/2013001/ article/11727-eng.htm].

99. Wolpowitz D, Gilchrest BA. The vitamin D questions: how much do you need and how should you get it? J Am Acad Dermatol. 2006;54(2):301-17.
100. Gilchrest BA. Sun exposure and vitamin D sufficiency. Am J Clin Nutr. 2008:88(2):570S-7S.

101. Vogeser M. Quantification of circulating 25-hydroxyvitamin D by liquid chromatography-tandem mass spectrometry. J Steroid Biochem Mol Biol. 2010;121(3-5):565-73.

102. Haddad JG, Chyu KJ. Competitive protein-binding radioassay for 25hydroxycholecalciferol. J Clin Endocrinol Metab. 1971;33(6):992-5.

103. Devgun MS, Johnson BE, Paterson CR. Ultraviolet radiation, weather and the blood levels of 25-hydroxyvitamin D. Clin Physiol Biochem. 1983;1(6):300-4.

104. Azizi E, Pavlotsky F, Vered I, Kudish Al. Occupational exposure to solar UVB and seasonal monitoring of serum levels of 25-hydroxy vitamin D3: a casecontrol study. Photochem Photobiol. 2009;85(5):1240-4.

105. Norsang G, Ma L, Dahlback A, Zhuoma C, Tsoja W, Porojnicu A, et al. The vitamin D status among Tibetans. Photochem Photobiol. 2009;85(4):1028-31.

106. Choi HS, Oh HJ, Choi H, Choi WH, Kim JG, Kim KM, et al. Vitamin D insufficiency in Korea-a greater threat to younger generation: the Korea National Health and Nutrition Examination Survey (KNHANES) 2008. J Clin Endocrinol Metab. 2011;96(3):643-51.

107. Oh MG, Han MA, Park J, Ryu SY, Choi SW. The prevalence of vitamin D deficiency among cancer survivors in a nationwide survey of the Korean population. PLoS One. 2015;10(6):e0129901.

108. Maeda SS, Kunii IS, Hayashi L, Lazaretti-Castro M. The effect of sun exposure on 25-hydroxyvitamin D concentrations in young healthy subjects living in the city of Sao Paulo, Brazil. Braz J Med Biol Res. 2007;40(12):1653-9.

109. Gulvady C, Pingle S, Shanbhag S. Incidence of vitamin B12 / D3 deficiency among company executives. Indian J Occup Environ Med. 2007;11(2):83-5.

110. Islam MZ, Shamim AA, Kemi V, Nevanlinna A, Akhtaruzzaman M, Laaksonen $M$, et al. Vitamin D deficiency and low bone status in adult female garment factory workers in Bangladesh. Br J Nutr. 2008;99(6):1322-9.

111. Vu LH, Whiteman DC, van der Pols JC, Kimlin MG, Neale RE. Serum vitamin $\mathrm{D}$ levels in office workers in a subtropical climate. Photochem Photobiol. 2011:87(3):714-20.

112. Cinar N, Harmanci A, Yildiz BO, Bayraktar M. Vitamin D status and seasonal changes in plasma concentrations of 25-hydroxyvitamin D in office workers in Ankara, Turkey. Eur J Intern Med. 2014;25(2):197-201.

113. Sharma N, Mangukiya K, Mali KL, Pareek UK, Sharma AK. Comparative study of the status of vitamin D3 in young office working women and housewives in Udaipur, Rajasthan. Int J Pharm Sci Res. 2015;6(5):2197-200.

114. Yosephin B, Khomsan A, Briawan D, Rimbawan R. Vitamin D plus calcium supplementation increased serum 25(OH)D on reproductive age women workers. Asian Pac J Trop Dis. 2015;5(11):877-80.

115. Kim HJ, Giovannucci E, Rosner B, Willett WC, Cho E. Longitudinal and secular trends in dietary supplement use: Nurses' Health Study and Health Professionals Follow-Up Study, 1986-2006. J Acad Nutr Diet. 2014;114(3):436-43.

116. Potula V, Henderson A, Kaye W. Calcitropic hormones, bone turnover, and lead exposure among female smelter workers. Arch Environ Occup Health 2005;60(4):195-204

117. Gonzalez-Padilla E, Soria Lopez A, Gonzalez-Rodriguez E, Garcia-Santana S, Mirallave-Pescador A. Groba Marco Mdel V, Saavedra P, Quesada Gomez JM, Sosa Henriquez M: High prevalence of hypovitaminosis D in medical students in Gran Canaria, Canary Islands (Spain). Endocrinol Nutr. 2011;58(6):267-73

118. Kaehler S, Baumgartner $H_{\text {, Jeske } M}$, Anliker M, Schennach $H$, Marschang $P$, et al. Prevalence of hypovitaminosis $D$ and folate deficiency in healthy young female Austrian students in a health care profession. Eur J Nutr. 2012;51(8):1021-31.

119. Al-Elq AH. The status of vitamin D in medical students in the preclerkship years of a Saudi medical school. J Family Community Med. 2012;19(2):100-4.

120. Manickam B, Washington T, Villagrana NE, Benjamin A, Kukreja S, Barengolts E. Determinants of circulating 25-hydroxyvitamin $D$ and bone mineral density in young physicians. Endocr Pract. 2012;18(2):219-26.

121. Zabihiyeganeh M, Adel Jahed S, Sarami S, Nojomi M. Hypovitaminosis D: Are medical students at risk? Int J Prev Med. 2014;5(9):1161-8.

122. Milovanovic OZ, Milovanovic JR, Djukic A, Matovic M, Lucic AT, Glumbic N, et al. Variation in vitamin D plasma levels according to study load of biomedical students. Acta Pol Pharm. 2015;72(1):213-5.

123. Orlandin Premaor M, Paludo P, Manica D, Paludo AP, Rossatto ER, Scalco R, et al. Hypovitaminosis $D$ and secondary hyperparathyroidism in resident physicians of a general hospital in southern Brazil. J Endocrinol Invest 2014. 2008;31(11):991-5. 
124. Singh S. Kumar, Prakash V, Tiwari S, Daliparthy D, Pratyush, Singh S, Jain P: Summer and winter prevalence of vitamin D deficiency of young resident doctors in North India. Nutr Diet. 2011;68(4):280-4.

125. Growdon AS, Camargo CA Jr, Clark S, Hannon M, Mansbach JM. Serum 25hydroxyvitamin D levels among Boston trainee doctors in winter. Nutrients. 2012;4(3):197-207.

126. Mendoza V, Villanueva MT, Vargas G, Gonzalez B, Halabe J, Simon J, et al. Vitamin $D$ deficiency among medical residents and its relationship with metabolic indices. Endocr Pract. 2013;19(1):59-63.

127. Ramirez-Vick M, Hernandez-Davila L, Rodriguez-Rivera N, LopezValentin M, Haddock L, Rodriguez-Martinez R, et al. Prevalence of vitamin D insufficiency and deficiency among young physicians at University District Hospital in San Juan, Puerto Rico. P R Health Sci J. 2015;34(2):83-8

128. Gann PH, Ma J, Hennekens CH, Hollis BW, Haddad JG, Stampfer MJ. Circulating vitamin $D$ metabolites in relation to subsequent development of prostate cancer. Cancer Epidemiol Biomark Prev. 1996; 5(2):121-6.

129. Goswami R, Gupta N, Goswami D, Marwaha RK, Tandon N, Kochuppillai N. Prevalence and significance of low 25-hydroxyvitamin D concentrations in healthy subjects in Delhi. Am J Clin Nutr. 2000;72(2):472-5.

130. Kramm H, Gangnon R, Jones AN, Linzer M, Hansen KE. The effect of physician workload on an educational intervention to increase vitamin $D$ screening. WMJ. 2010;109(3):136-41.

131. Lee JE, Li H, Chan AT, Hollis BW, Lee IM, Stampfer MJ, et al. Circulating levels of vitamin D and colon and rectal cancer: the Physicians' Health Study and a meta-analysis of prospective studies. Cancer Prev Res (Phila Pa). 2011;4(5):735-43.

132. Haliloglu $O$, Kasaci T, Yavuz D. Seasonal vitamin D status and endothelial function in healthcare workers. Turk J Med Sci. 2016:46(1):72-8.

133. Munter G, Levi-Vineberg T, Sylvetsky N. Vitamin D deficiency among physicians: a comparison between hospitalists and community-based physicians. Osteoporos Int. 2015;26(6):1673-6.

134. Platz EA, Hankinson SE, Hollis BW, Colditz GA, Hunter DJ, Speizer FE, et al. Plasma 1,25-dihydroxy- and 25-hydroxyvitamin D and adenomatous polyps of the distal colorectum. Cancer Epidemiol Biomark Prev. 2000;9(10):1059-65.

135. Eliassen AH, Spiegelman D, Hollis BW, Horst RL, Willett WC, Hankinson SE. Plasma 25- hydroxyvitamin D and risk of breast cancer in the Nurses' Health Study II. Breast Cancer Res. 2011;13(3):R50.

136. Hattapornsawan $Y$, Pangsuwan S, Ongphiphadhanakul B, Udomsubpayakun $U$. Prevalence of vitamin D deficiency in nurses at the Royal Irrigation Hospital. J Med Assoc Thail. 2012;95(12):1569-74.

137. Wallingford SC, Jones G, Kobayashi LC, Grundy A, Miao Q, Tranmer J, et al. UV and dietary predictors of serum 25-hydroxyvitamin D concentrations among young shift-working nurses and implications for bone density and skin cancer. Public Health Nutr. 2014;17(4):772-9.

138. Wang J, Eliassen AH, Spiegelman D, Willett WC, Hankinson SE. Plasma free 25-hydroxyvitamin D, vitamin D binding protein, and risk of breast cancer in the Nurses' Health Study II. Cancer Causes Control. 2014;25(7):819-27.

139. Bertrand KA, Rosner B, Eliassen AH, Hankinson SE, Rexrode KM, Willett W, et al. Premenopausal plasma 25-hydroxyvitamin D, mammographic density, and risk of breast cancer. Breast Cancer Res Treat. 2015;149(2):479-87.

140. Alavi NM, Madani M, Taghizadeh M, Sharif MR. Vitamin D supplementation in female nurses: The effects on serum 25-hydroxyvitamin D, and nonspecific musculoskeletal pain. J Musculosketeletal Res. 2015;18(2):1550008. $-1-1550008-9$

141. Nakamura K, Nashimoto M, Matsuyama S, Yamamoto M. Low serum concentrations of 25-hydroxyvitamin D in young adult Japanese women: a cross sectional study. Nutrition. 2001;17(11-12):921-5.

142. Platz EA, Leitzmann MF, Hollis BW, Willett WC, Giovannucci E. Plasma 1,25dihydroxy- and 25-hydroxyvitamin D and subsequent risk of prostate cancer. Cancer Causes Control. 2004;15(3):255-65.

143. Arya V, Bhambri R, Godbole MM, Mithal A. Vitamin D status and its relationship with bone mineral density in healthy Asian Indians. Osteoporos Int. 2004;15(1):56-61.

144. Hanwell HE, Vieth R, Cole DE, Scillitani A, Modoni S, Frusciante V, et al. Sun exposure questionnaire predicts circulating 25-hydroxyvitamin D concentrations in Caucasian hospital workers in southern Italy. J Steroid Biochem Mol Biol. 2010;121(1-2):334-7.
145. Plotnikoff GA, Finch MD, Dusek JA. Impact of vitamin D deficiency on the productivity of a health care workforce. J Occup Environ Med. 2012;54(2): 117-21.

146. Porojnicu AC, Moroti-Constantinescu R, Laslau A, Lagunova Z, Dahlback A Hristea $A$, et al. Vitamin D status in healthy Romanian caregivers and risk of respiratory infections. Public Health Nutr. 2012;15(11):2157-62.

147. Gannage-Yared MH, Helou E, Zaraket V, Abi Akl S, Antonios L, Moussalli ML, et al. Serum 25-hydroxyvitamin D in employees of a Middle Eastern university hospital. J Endocrinol Investig. 2014;37(6):541-6.

148. Skarphedinsdottir SJ, Sigurdsson MI, Coursin DB, Head DE, Springman SR, Wang $S$, et al. Vitamin D deficiency in anesthesia department caregivers at the end of winter. Acta Anaesthesiol Scand. 2014;58(7):802-6.

\section{Submit your next manuscript to BioMed Central and we will help you at every step:}

- We accept pre-submission inquiries

- Our selector tool helps you to find the most relevant journal

- We provide round the clock customer support

- Convenient online submission

- Thorough peer review

- Inclusion in PubMed and all major indexing services

- Maximum visibility for your research

Submit your manuscript at www.biomedcentral.com/submit
Biomed Central 